Bundesgesundheitsbl 2015 · 58:1151-1170

DOI 10.1007/s00103-015-2234-2

Online publiziert: 28. September 2015

c) Springer-Verlag Berlin Heidelberg 2015

CrossMark

\title{
Infektionsprävention im Rahmen der Pflege und Behandlung von Patienten mit übertragbaren Krankheiten
}

\author{
Empfehlung der Kommission für \\ Krankenhaushygiene und Infektionsprä- \\ vention (KRINKO) beim Robert Koch-Institut
} Leitungen von medizinischen Einrichtungen und Krankenhäusern, Heimen und Betreuungseinrichtungen, in denen Patienten oder Bewohner untergebracht

\footnotetext{
1 In dieser Empfehlung werden zur besseren Lesbarkeit die Bezeichnungen Patient, Beschäftigter oder Besucher genutzt, unabhängig davon ob es sich um männliche oder weibliche Personen handelt.
}

und gepflegt werden, bei denen akut Infektionserkrankungen auftreten können.

Allerdings kann die Umsetzung einer Reihe der aufgeführten Maßnahmen auch in ambulanten medizinischen Einrichtungen bzw. Einrichtungen des Gesundheitswesens notwendig sein, da Patienten mit Infektionskrankheiten auch dort diagnostiziert und behandelt werden können. In diesen Fällen sind die Empfehlungen an den durchgeführten medizinischen und pflegerischen Maßnahmen auszurichten und nicht an den Ort der Durchführung.

Nicht berücksichtigt werden Maßnahmen bei Besiedelung mit epidemiologisch relevanten Erregern, z. B. multiresistenten Erregern und Maßnahmen bei Ausbrüchen von Infektionserkrankungen. Erkrankungen der Schutzstufe 4 (z. B. hämorrhagisches Fieber, wie Ebola-, Lassa-, Marburg-, Krim-Kongo-Hämorrhagisches-Fieber und Pocken) werden ebenfalls nicht berücksichtigt, da $\mathrm{Pa}$ tienten mit solchen Erkrankungen nur in Sonderisolierstationen behandelt werden sollen $[2,3]$.

\subsection{Bezug zu anderen Empfehlungen}

gen zur Händehygiene [4], zur Flächendesinfektion [5], zur Aufbereitung von Medizinprodukten [6] sowie in den Empfehlungen zur Hygiene bei einzelnen medizinischen Maßnahmen, z. B. zur Hygiene bei Punktionen und Injektionen [7] aufgeführt. Auf die besondere Infektionsgefährdung immunsupprimierter Patienten [8] oder von intensivmedizinisch behandelten Frühgeborenen [9] wird in den entsprechenden Empfehlungen eingegangen.

Empfehlungen zur Prävention der Übertragung multiresistenter Erreger sind z. B. in den Empfehlungen zur Prävention und Kontrolle von Methicillin-resistenten Staphylococcus aureus-Stämmen (MRSA) in Krankenhäusern und anderen medizinischen Einrichtungen [10] und Hygienemaßnahmen bei Infektionen oder Besiedlung mit multiresistenten gramnegativen Stäbchen (MRGN) [11] zusammengestellt.

Diese Empfehlungen werden durch die vorliegende Empfehlung nicht ersetzt. Im Sinne einer Darstellung der Basishygiene als Bündel und einem erleichterten Umgang mit dem Dokument werden jedoch einige Maßnahmen hervorgehoben.

\subsection{Kategorisierung}

Viele Empfehlungen zur Infektionsprävention stellen allgemeine, nicht erregerspezifische, immer anzuwendende Maßnahmen dar, welche die Übertragung von Erregern, wie sie bei allen Patientenkontakten auftreten können, verhindern sollen. Diese sogenannten Basishygienemaßnahmen werden z. B. in den Empfehlun-
Die Elemente der Basishygiene sind in anderen KRINKO-Empfehlungen (z. B. zur Händehygiene, zur Reinigung und Desinfektion von Flächen oder zur Aufbereitung von Medizinprodukten) genauer dargestellt. Die grundlegenden Maßnahmen werden lediglich synoptisch darge- 


\begin{tabular}{|c|c|}
\hline \multicolumn{2}{|l|}{ Glossar } \\
\hline Disposition & $\begin{array}{l}\text { Genetisch bedingte oder erworbene Anfälligkeit für die Ausbildung von } \\
\text { Krankheiten }\end{array}$ \\
\hline Exposition & $\begin{array}{l}\text { Ausgesetzt sein von Lebewesen gegenüber schädigenden Umweltein- } \\
\text { flüssen wie Krankheitserregern }\end{array}$ \\
\hline Immunität & $\begin{array}{l}\text { Fähigkeit des Organismus, bestimmte Pathogene ohne Symptome zu } \\
\text { eliminieren }\end{array}$ \\
\hline Infektionsdosis & Anzahl an Pathogenen einer Spezies, die ein Wirt aufgenommen hat \\
\hline Infektiosität & Fähigkeit eines Pathogens, einen Wirt zu infizieren \\
\hline Kontagiosität & $\begin{array}{l}\text { Übertragungsfähigkeit eines Pathogens in Abhängigkeit vom erreger- } \\
\text { typischen Infektionsweg }\end{array}$ \\
\hline Kontagionsindex & $\begin{array}{l}\text { Anteil einer nicht-immunen Population, bei dem es nach Kontakt mit } \\
\text { einem Krankheitserreger zu einer Infektion kommt }\end{array}$ \\
\hline Letalität & $\begin{array}{l}\text { Sterblichkeit einer Erkrankung, berechnet als das Verhältnis der Todes- } \\
\text { fälle zur Anzahl der an einer spezifischen Krankheit Erkrankten }\end{array}$ \\
\hline Manifestationsindex & $\begin{array}{l}\text { Wahrscheinlichkeit mit der eine mit einem Erreger infizierte Person } \\
\text { erkennbar erkrankt }\end{array}$ \\
\hline $\begin{array}{l}\text { Minimale Infektions- } \\
\text { dosis }\end{array}$ & $\begin{array}{l}\text { Mindestanzahl an Pathogenen einer Spezies, die notwendig ist, um eine } \\
\text { Infektion auszulösen }\end{array}$ \\
\hline Pathogenität & $\begin{array}{l}\text { grundsätzliche Fähigkeit von infektiösen Organismen, bei einem Wirt zu } \\
\text { einer Erkrankung zu führen }\end{array}$ \\
\hline Vektor & $\begin{array}{l}\text { lebender Organismus, der Krankheitserreger von einem infizierten Tier } \\
\text { oder einem infizierten Menschen auf einen Menschen überträgt }\end{array}$ \\
\hline Virulenz & Maß für die Pathogenität \\
\hline $\begin{array}{l}\text { Für weitere Definitionen } \\
\text { ter - Definitionen - Inter }\end{array}$ & $\begin{array}{l}\text { auf das Fachwörterbuch Infektionsschutz und Infektionsepidemiologie. Fachwör- } \\
\text { ationen verwiesen [1]. }\end{array}$ \\
\hline
\end{tabular}

stellt und wurden bereits in anderen bestehenden Empfehlungen ausführlich kommentiert und ggf. kategorisiert. Daher wurde in dieser Empfehlung auf eine Kategorisierung verzichtet.

Über die Basishygiene hinaus werden konkrete Maßnahmen in der Tabelle zusammengefasst, in der die bei den jeweiligen Infektionen gebotenen, über die Basishygiene hinausgehenden Präventionsmaßnahmen in einer Übersicht dargestellt werden. Die dort aufgeführten Maßnahmen leiten sich nicht aus klinischen Studien, sondern primär aus den Transmissionswegen und Eigenschaften der jeweiligen Erreger ab. Diese Informationen finden sich jeweils in den Ratgebern für Ärzte, herausgegeben vom Robert Koch-Institut (RKI) und abrufbar auf der Homepage des RKI (www.rki.de). befasst sich mit der Ursache, der Verteilung, den Risikofaktoren und der Kontrolle von übertragbaren Erkrankungen. Um infektiöse Erkrankungen kontrollieren zu können, ist das Wissen über die Ursache, d. h. den Erreger und seine Eigenschaften, notwendig. Die Eigenschaften des Erregers beeinflussen die Wahrscheinlichkeit, ob dieser von Mensch zu Mensch oder via Vektor übertragen wird und/oder sogar stabil in der Umwelt überlebt und von dort von seinem Wirt aufgenommen werden kann. Erreger, die direkt von Mensch zu Mensch übertragen werden, sind häufig sehr viel empfindlicher gegenüber Umwelteinflüssen als Erreger, die regelmäßig in der Umgebung gefunden werden.

Grundsätzlich können verschiedene Arten von Übertragungswegen unterschieden werden (siehe Kapitel 3.1)

- Parenterale Übertragung

- Kontaktübertragung (direkt oder indirekt)

- Tröpfchenübertragung

- Aerogene Übertragung

- Vektorassoziierte Übertragung

Dabei muss bedacht werden, dass für die meisten Erreger mehrere Übertragungswege möglich sind [13-18].

\subsection{Präventionsmaßnahmen}

Auch asymptomatische Personen können mit von Mensch zu Mensch übertragbaren Infektionserregern besiedelt oder infiziert sein. Daher müssen im Behandlungsalltag stets im Umgang mit allen $\mathrm{Pa}$ tienten bestimmte grundlegende Präventionsmaßnahmen eingehalten werden, die sowohl dem Schutz anderer Patienten als auch dem Schutz des Personals vor einer Übertragung dienen. Diese immer einzuhaltenden Maßnahmen werden als Maßnahmen der Basishygiene bezeichnet und sind nachfolgend für die einzelnen Schutzmaßnahmen zusammenfassend aufgeführt. Im Zusammenhang mit medizinischen Maßnahmen dient die Basishygiene der Prävention nosokomialer Infektionen. 


\section{Basishygiene}

\subsection{Händehygiene}

Die meisten Krankheitserreger können über kontaminierte Hände übertragen werden. Die nicht desinfizierten Hände der Beschäftigten und ggf. der Patienten sind ein wichtiges Übertragungsvehikel der meisten Krankheitserreger von einem Patienten auf den anderen. Die Händedesinfektion dient daher nicht nur dem Schutz der Beschäftigten, sondern sie ist eine der wichtigsten Maßnahmen zur Verhütung von nosokomialen Infektionen. Indikationen für die Händedesinfektion entstehen vor und nach direktem Kontakt mit dem Patienten, vor aseptischen Tätigkeiten, nach Kontamination (Kontakt mit Blut, Sekreten oder Exkreten), nach Kontakt mit der Patientenumgebung, sowie nach Ablegen von Einmalhandschuhen. Einzelheiten hierzu sind der KRINKO-Empfehlung zur Händehygiene zu entnehmen [4].

\subsection{Barrieremaßnahmen}

Die persönliche Schutzausrüstung (PSA) bildet eine mechanische Barriere zwischen dem Träger und seiner Umgebung. Daher dient ihr Einsatz nicht nur dem Schutz des Personals sondern auch dazu (bei sachgerechter Anwendung und Entsorgung), die Weiterverbreitung von Krankheitserregern zu verhindern [19]. Als zentrales Element des Arbeitsschutzes wurde bereits am 30. November 1989 die europäische Richtlinie über die Mindestvorschriften für Sicherheit und Gesundheitsschutz bei Benutzung persönlicher Schutzausrüstung durch Arbeitnehmer bei der Arbeit erlassen (ABI. EG Nr. L 393 S. 18). In nationales Recht wurde diese Richtlinie umgesetzt durch die PSABenutzungsverordnung vom 4. Dezember 1996 (BGBl. I S. 1841).

Mit dieser Vorbemerkung ist der Hinweis verbunden, dass die nachfolgenden Aussagen und Empfehlungen auch auf den Schutz der Patienten abzielen, wegen des fachlichen Bezugs und je nach Fragestellung aber primär der betriebsärztliche Dienst Ansprechpartner zur sachgerechten Anwendung von PSA sein sollte.

\subsubsection{Einmalhandschuhe}

Als nicht-sterile Einmalhandschuhe dienen mindestens medizinische Handschuhe, die entsprechend TRBA 250 nach DIN EN 455 „Medizinische Handschuhe zum einmaligen Gebrauch“ hinsichtlich der Dichtheit ein akzeptiertes Qualitätsniveau (AQL) von $<1,5$ aufweisen [2, 20]. Da im medizinischen Bereich häufiger Kontakt zu Chemikalien wie Desinfektionsmitteln besteht, kann es sinnvoll sein Schutzhandschuhe zu verwenden, die zunächst den allgemeinen Anforderungen der DIN EN 420 (Schutzhandschuhe) entsprechen und zudem nach DIN EN 374 Teil 1 „Schutzhandschuhe gegen Chemikalien und Mikroorganismen "Chemikalienbeständigkeit aufweisen [21, 22].

Nicht-sterile Einmalhandschuhe werden zur Vermeidung der Kontamination der Hände des Personals verwendet, wenn direkter Kontakt mit Blut, Sekreten, Exkreten, Schleimhäuten, oder nicht intakter Haut zu erwarten ist. Hierzu gehört auch der Handkontakt zu Geräten, Instrumenten oder Oberflächen, die sichtbar oder wahrscheinlich mit bestimmten Krankheitserregern kontaminiert sind. Einmalhandschuhe haben sich als effektiv erwiesen, die Übertragung von Erregern, die durch Kontakt weiterverbreitet werden, zu reduzieren [23, 24]. Einmalhandschuhe stellen aber nur eine relative und keine absolute Barriere für Krankheitserreger dar. Das Tragen von Einmalhandschuhen ersetzt nicht die Notwendigkeit zur Händedesinfektion [25]. Nach Ablegen der Einmalhandschuhe ist stets eine Händedesinfektion erforderlich, da es durch Leckagen und beim Prozess des Ablegens der Handschuhe zur Kontamination der Hände kommen kann. Einmalhandschuhe werden somit nicht anstelle, sondern zusätzlich zur Händedesinfektion eingesetzt [26].

Einige Studien haben untersucht, ob das generelle Tragen von keimarmen Einmalhandschuhen bei jedem Patientenkontakt (,universal gloving') einen Einfluss auf nosokomiale Infektionsraten hat [25-28]. Diese Studien sind methodisch sehr heterogen und ihre Ergebnisse uneinheitlich.

Beim Umgang mit nicht-sterilen Einmalhandschuhen ist darauf zu achten, dass sie so gelagert und aus der Verpackung entnommen werden, dass sie nicht mit potentiell pathogenen Mikroorganismen konta- miniert werden. Weitere Einzelheiten zum Umgang mit nicht-sterilen Einmalhandschuhen sind der KRINKO-Empfehlung zur Händehygiene zu entnehmen [4].

\subsubsection{Schürzen und Schutzkittel}

Arbeitskleidung ist eine Kleidung, die anstelle oder in Ergänzung der Privatkleidung bei der Arbeit getragen wird. Zur Arbeitskleidung zählt auch Berufs- bzw. Bereichskleidung. Die Rolle der Kleidung bei der Übertragung von Infektionserregern wird kontrovers diskutiert. Einige Mikroorganismen sind in der Lage in der unbelebten Umgebung (auch auf textilen Oberflächen) zu überleben [29-31]. Vor allem Handkontaktstellen der Kleidung haben sich als kontaminiert erwiesen [32]. Der Nachweis einer Kontamination der Bereichs-/Arbeitskleidung von Mitarbeitern in der direkten Patientenbetreuung und der ,weißen Kittel' von Ärztinnen und Ärzten mit Krankheitserregern, beweist nicht die nosokomiale Übertragung pathogener (zum Teil multiresistenter) Infektionserreger auf weitere Patienten [33]. Wiener-Well et al. untersuchten anhand von 238 Proben die bakterielle Kontamination der Arbeitskleidung von Ärzten $(n=60)$ und Pflegepersonal $(n=75)$ (abdominelle Zone, Ärmelenden und Kitteltaschen) [32]. Von den Teilnehmenden gaben $58 \%$ an, die Arbeits- bzw. Bereichskleidung täglich zu wechseln; $77 \%$ empfanden ihren Kittel als ziemlich oder sehr sauber. Bei 85 Teilnehmenden $(63 \% n=135)$ wurden in den Proben pathogene Bakterien nachgewiesen (50\% aller entnommenen Proben waren positiv, $n=238$ ). Beim Pflegepersonal ließen sich in $14 \%$ aller Proben Erreger mit speziellen Antibiotikaresistenzen nachweisen, der entsprechende Anteil bei den Ärzten lag bei $6 \%$. Diese Untersuchung von Wiener-Well et al. [32] steht in einer Reihe von methodisch gut ausgeführten Arbeiten, in denen die Kontamination von Bereichskleidung durch potentiell pathogene, teils auch multiresistente Infektionserreger während der Arbeit am Patienten beschrieben wird [34-40]. Diese Befunde sollten auch in Überlegungen zum Ablauf der hygienischen Händedesinfektion vor Patientenkontakt einfließen. So macht es wenig Sinn, sich erst die Hände zu desinfizieren und dann das Stethoskop 
zur Untersuchung des Patienten aus der (möglicherweise kontaminierten) Kitteltasche zu ziehen [39, 41-45].

Wiener-Well et al. [32] schlagen vor, dass Beschäftigte, die eng am Patienten arbeiten, ihre Arbeits-/Bereichskleidung täglich wechseln und situationsbedingt zusätzlich patientenbezogene Schutzkittel/ Schürzen über der Bereichskleidung tragen. Kurzärmelige Kasaks ohne Knopfleisten sind auch für Ärztinnen und Ärzte mit engem Patientenkontakt als Bereichskleidung besonders praktikabel, da sie nicht auf- und zugeknöpft werden müssen und es keine Ärmel oder Bündchen gibt, die man kontaminieren könnte. Das National Institute for Health and Care Excellence (NICE, Großbritannien) hat in seinen Empfehlungen (Prevention and control of healthcare-associated infections in primary and community care [46]) die „bare below elbows“-Empfehlung herausgegeben, nach der neben Verzicht auf Schmuck an Händen und Unterarmen das Tragen kurzärmeliger Kleidung empfohlen wird.

Auswirkungen der Art der Kleidung mit langen oder kurzen Ärmeln auf die Qualität der Händedesinfektion konnten nicht gezeigt werden [47-50]. Zusammenfassend sind die vorliegenden Daten hinsichtlich des Zieles der Vermeidung von Infektionen jedoch nicht ausreichend für eine Empfehlung.

Patientenbezogene Schürzen oder Schutzkittel werden über und zusätzlich zur Bereichs-/Arbeitskleidung getragen, wenn im Kontakt zu einem Patienten oder zu seiner unmittelbaren Umgebung eine Kontamination der Kleidung mit Infektionserregern wahrscheinlich ist. Während Schürzen ärmellos und hinten offen sind, sind Schutzkittel langärmelig (mit Bündchen) und rückenbedeckend. Die Auswahl geeigneter Schutzkittel/Schürzen richtet sich nach der vorgesehen Tätigkeit, dem erwarteten Kontaminationsrisiko und dem Risiko der Durchfeuchtung. Im medizinischen Bereich sollten nur Schürzen und Schutzkittel zum Einsatz kommen, die den Anforderungen an Medizinprodukte und Schutzkleidung genügen (z. B. CE Kennzeichnung (Gruppe 3) oder DIN EN 14126 [51]).

In diesem Zusammenhang wird darauf hingewiesen, dass Schutzkleidung für Mitarbeiter in der direkten Patientenbetreu- ung vom Arbeitgeber in ausreichender Stückzahl gestellt werden muss. Arbeitskleidung von Beschäftigten in der direkten Patientenversorgung ist wie oben ausgeführt, häufig mikrobiologisch kontaminiert. Sofern sie kontaminiert ist, muss sie mit einem desinfizierenden Verfahren mit nachgewiesener Wirksamkeit aufbereitet werden [2]. Es hat sich daher als praktikabel erwiesen, Beschäftigten in der direkten Patientenversorgung Arbeitskleidung in ausreichender Stückzahl, z. B. für den täglichen Wechsel zur Verfügung zu stellen, und diese generell mit einem desinfizierenden Verfahren mit nachgewiesener Wirksamkeit aufzubereiten.

\subsubsection{Mund-Nasen-Schutz und Augenschutz}

Im Rahmen des Schutzes der Patienten kann ein Mund-Nasen-Schutz während bestimmter aseptischer Prozeduren den Patienten vor Mikroorganismen aus dem Mund-Rachen-Raum der Beschäftigten schützen, die beim Sprechen und Husten abgegeben werden.

Die Schleimhäute von Augen, Mund und Nase sind potentielle Eintrittspforten für Krankheitserreger [52-55]. Ein MundNasen-Schutz kann Beschäftigte bei engen Patientenkontakten vor verspritztem Blut oder Spritzern anderer Körpersekrete schützen und ist ein wirksamer Schutz vor Berührung von Mund und Nase mit kontaminierten Händen. Um auch die Augen zu schützen müssen in den genannten Fällen zusätzlich Schutzbrillen oder ggf. Gesichtsmasken getragen werden $[56,57]$.

\section{Die Kommission empfiehlt}

- nicht-sterile Einmalhandschuhe zu tragen, wenn die Wahrscheinlichkeit des Kontaktes mit Blut, Sekreten, Exkreten oder wahrscheinlich kontaminierten Flächen besteht.

- das Tragen einer Schürze oder eines Schutzkittels, um Arbeitskleidung bei Eingriffen oder Pflegemaßnahmen vor direktem Kontakt mit Blut, Sekreten, Exkreten oder mit anderen kontaminierten Materialien zu schützen.

- das Tragen von Mund-Nasen-Schutz und Schutzbrille oder eines Gesichtsschutzschildes, wenn mit Verspritzen von Blut, Sekreten oder Exkreten zu rechnen ist.

\subsection{Flächendesinfektion}

Oberflächen in der Patientenumgebung müssen so behandelt werden, dass von ihnen kein Infektionsrisiko ausgehen kann. Dafür hat es sich als sinnvoll erwiesen, die Flächen in der Umgebung des Patienten nach dem möglichen Übertragungsrisiko zu klassifizieren und laufende Desinfektions- und Reinigungsmaßnahmen entsprechend im Hygieneplan festzulegen [5]. Flächen, die als Risikoflächen mit häufigem Hand- oder Hautkontakt klassifiziert sind, müssen mindestens einmal täglich, bei sichtbarer Kontamination sofort, desinfizierend aufbereitet werden. Bei diesen Flächen ist die sogenannte „Sichtreinigung“, d. h. die alleinige Beseitigung sichtbarer Verschmutzungen, nicht ausreichend.

\section{Die Kommission empfiehlt}

- Risikoflächen mit häufigem Handund Hautkontakt mindestens täglich so aufzubereiten, dass keine Übertragungsgefahr davon ausgeht. Bei sichtbarer Kontamination muss die Aufbereitung sofort erfolgen.

\subsection{Aufbereitung von Medizinprodukten}

Zur Prävention der Übertragung von Mikroorganismen durch Medizinprodukte sind die Empfehlungen der KRINKO und des BfArM „Anforderungen an die Hygiene bei der Aufbereitung von Medizinprodukten“ zu berücksichtigen [6].

\subsection{Abfallentsorgung}

Für die Abfallentsorgung wird auf die Vollzugshilfe zur Entsorgung von Abfällen aus Einrichtungen des Gesundheitsdienstes der Bund/Länder-Arbeitsgemeinschaft Abfall (LAGA) verwiesen [58].

\subsection{Umgang mit Wäsche}

Über Wäsche können Infektionserreger sowohl auf Patienten als auch auf Personal übertragen werden [59]. 


\subsubsection{Bettenhygiene und Bettwäsche}

Jeder Patient muss im Krankenhaus ein aufbereitetes, desinfiziertes Bett erhalten, welches mit desinfizierten Inletts (Bettdecke und Kopfkissen), bezogen mit sauberer Wäsche versehen ist [60]. Nach Risikobewertung kann ggf. auf die desinfizierende Aufbereitung der Inletts zwischen zwei Patienten verzichtet werden.

Untersuchungen zur notwendigen Frequenz des Wäschewechsels liegen nicht vor. Die Wäsche muss bei sichtbarer Verschmutzung sofort gewechselt werden, ansonsten hat sich ein mindestens einmal wöchentlicher Wechsel der Bettwäsche bewährt.

\subsubsection{Wäscheentsorgung, -aufbereitung und -versorgung}

Gebrauchte und kontaminierte Wäsche muss in der medizinischen Einrichtung so gesammelt und transportiert werden, dass von ihr keine Infektions- oder Kontaminationsgefahr ausgeht. Sie soll unmittelbar im Arbeitsbereich in ausreichend widerstandsfähigen und dichten sowie eindeutig gekennzeichneten Behältnissen gesammelt werden. Eine Abstimmung zwischen den Arbeitsbereichen, in denen die Wäsche anfällt, und der Wäscherei, ist zur richtigen Sammlung und Kennzeichnung erforderlich [2].

Saubere Wäsche muss frei von Krankheitserregern und keimarm sein. Dies ist durch Anwendung von entsprechenden desinfizierenden Waschverfahren mit nachgewiesener Wirksamkeit zu gewährleisten, unabhängig davon, ob die Wäsche innerhalb oder außerhalb des Krankenhauses aufbereitet wird [61]. Die Wäsche muss so behandelt werden, dass die Patienten nicht gegenüber Waschmittelrückständen exponiert werden.

\subsubsection{Bekleidung für Personal und Patienten}

Wird Bekleidung (für Personal oder Patienten) im Krankenhaus aufbereitet, so müssen auch hierfür geeignete Waschverfahren verwendet werden, mit denen sichergestellt ist, dass die Bekleidung anschließend keimarm und frei von Krankheitserregern ist.

Nach Abschluss des Waschvorganges muss die saubere Wäsche so transportiert und gelagert werden, dass eine Rekontamination vermieden wird.

\section{Die Kommission empfiehlt}

- jedem Patienten bei stationärer Aufnahme ein desinfizierend aufbereitetes, mit desinfizierten Inletts und sauberer Wäsche bezogenes Bett zur Verfügung zu stellen.

- die Wäsche bei sichtbarer Verunreinigung sofort zu wechseln.

- gebrauchte und kontaminierte Wäsche so zu sammeln und zu transportieren, dass von ihr keine Infektionsoder Kontaminationsgefahr ausgeht.

- Wäsche so aufzubereiten und zu lagern, dass sie dem Patienten sauber, keimarm und frei von Rückständen zur Verfügung gestellt wird.

- jegliche Aufbereitung von Wäsche aus medizinischen Einrichtungen mit desinfizierenden, regelmäßig überprüften Verfahren mit nachgewiesener Wirksamkeit durchzuführen.

\subsection{Umgang mit Geschirr}

Beschreibungen von Ausbrüchen oder Übertragungen nosokomialer Infektionen durch Patienten-Geschirr konnten durch Medline-Abfragen [62] nicht identifiziert werden. Dennoch steht außer Frage, dass jeder Patient Speisen und Getränke auf bzw. in sauberem und keimarmem Geschirr erhält. Die Aufbereitung von Geschirr soll bevorzugt maschinell erfolgen. Es sind die entsprechenden lebensmittelhygienischen Vorgaben [63] zu berücksichtigen.

Bei Umgang mit und Transport von benutztem Geschirr muss darauf geachtet werden, dass von dem Geschirr keine Kontaminationsgefahr ausgeht.

\subsection{Aufklärung und Schulung von Patienten und deren Besuchern}

Bisher gibt es nur wenige Untersuchungen inwieweit Patienten selbst zur Übertragung von Erregern in medizinischen Einrichtungen beitragen [64]. Dennoch möchte zum Beispiel die Mehrzahl der Patienten, die mit multiresistenten Erregern besiedelt sind, über Infektionsrisiken informiert werden und selbst Verantwortung für die Infektionsprävention über- nehmen [65]. Entsprechende Schulungsprogramme können Bewusstsein und Verhalten der Patienten zur Hygiene verändern und damit zur Infektionsprävention beitragen [66].

\section{Die Kommission empfiehlt}

- Patienten und deren Besucher zu persönlichen Maßnahmen der Hygiene aufzuklären.

\subsection{Art der Unterbringung}

Die meisten medizinischen oder Pflegeeinrichtungen haben nur einen begrenzten Anteil an Einzelzimmern, so dass verschiedene konkurrierende Bedürfnisse bei der Unterbringung der Patienten berücksichtigt werden müssen. In Einzelzimmern sollten vor allem solche Patienten behandelt werden, von denen ein erhöhtes Übertragungsrisiko ausgeht, z. B. auch Patienten, denen adäquates hygienisches Verhalten nicht möglich ist. In solchen Fällen sollen die Einzelzimmer über ein eigenes Patientenbad verfügen.

\section{Zu den Maßnahmen der Basis- hygiene gehören zusammen- gefasst}

Händehygiene:

- Durchführung der Händedesinfektion vor und nach direktem Kontakt mit dem Patienten, vor aseptischen Tätigkeiten, nach Kontamination (Kontakt mit Blut, Sekreten oder Exkreten), nach Kontakt mit der Patientenumgebung, sowie nach Ablegen von Einmalhandschuhen (siehe auch entsprechende Empfehlung der KRINKO)

Barrieremaßnahmen:

- Tragen nicht-steriler Einmalhandschuhe, wenn die Wahrscheinlichkeit des Kontaktes mit Blut, Sekreten, Exkreten oder wahrscheinlich kontaminierten Flächen besteht

- Tragen einer Schürze oder eines Schutzkittels, um Arbeitskleidung bei Eingriffen oder Pflegemaßnahmen vor direktem Kontakt mit Blut, Sekreten, Exkreten oder mit anderen kontaminierten Materialien zu schützenTragen von Mund-NasenSchutz und Schutzbrille oder eines Gesichtsschutzschildes, wenn mit Verspritzen von Blut oder Sekreten zu rechnen ist 
Flächendesinfektion:

- Aufbereitung von Risikoflächen mit häufigem Hand- und Hautkontakt mindestens täglich so, dass keine Übertragungsgefahr davon ausgeht; bei sichtbarer Kontamination muss die Aufbereitung sofort erfolgen (siehe auch entsprechende Empfehlung der KRINKO)

Aufbereitung von Medizinprodukten:

- Aufbereitung von Medizinprodukten entsprechend den Empfehlungen der KRIN$\mathrm{KO}$ und des BfArM „Anforderungen an die Hygiene bei der Aufbereitung von Medizinprodukten" (siehe entsprechende Empfehlung)

Abfallentsorgung:

- Abfallentsorgung entsprechend der Vollzugshilfe zur Entsorgung von Abfällen aus Einrichtungen des Gesundheitsdienstes der Bund/Länder-Arbeitsgemeinschaft Abfall (LAGA)

Bettenhygiene und Bettwäsche:

- Jedem Patienten bei stationärer Aufnahme ein desinfizierend aufbereitetes, mit desinfizierten Inletts und sauberer Wäsche bezogenes Bett zur Verfügung zu stellen

- Die Wäsche bei sichtbarer Verunreinigung sofort zu wechseln

Wäscheentsorgung, -aufbereitung und -versorgung und Bekleidung für Personal und $\mathrm{Pa}$ tienten:

- Gebrauchte und kontaminierte Wäsche so zu sammeln und zu transportieren, dass von ihr keine Infektions- oder Kontaminationsgefahr ausgeht

- Wäsche so aufzubereiten und zu lagern, dass sie dem Patienten sauber, keimarm und frei von Rückständen zur Verfügung gestellt wird

- Jegliche Aufbereitung von Wäsche aus medizinischen Einrichtungen mit desinfizierenden, regelmäßig überprüften Verfahren mit nachgewiesener Wirksamkeit durchzuführen Umgang mit Geschirr:

- Jedem Patienten Speisen und Getränke auf bzw. in sauberem und keimarmen (desinfiziertem) Geschirr zu reichen

Aufklärung und Schulung von Patienten und deren Besuchern:

- Patienten und deren Besucher zu persönlichen Maßnahmen der Hygiene aufzuklären Art der Unterbringung:

- Patienten, von denen ein erhöhtes Übertragungsrisiko ausgeht in Einzelzimmern zu behandeln

\section{Erweiterte, über die Basishygiene hinausgehende Maßnahmen}

Bereits bei begründetem Verdacht auf eine übertragbare Erkrankung sollen entsprechend der Verdachtsdiagnose angepasste Schutzmaßnahmen eingeleitet werden.

Bei der Festlegung von über die Basishygiene hinausgehenden Schutzmaßnahmen für Personal einschließlich Transportpersonal, Mitpatienten und Besuchern, ist vorab eine Einschätzung des Übertragungsrisikos von Infektionserregern sinnvoll. Hierbei ist, neben Virulenz und Infektionsdosis von Erregern, die Kenntnis des Übertragungsweges und der Exposition sowie der Disposition und Immunität des zu Schützenden von entscheidender Bedeutung.

Verschiedene Risikofaktoren beeinflussen die Wahrscheinlichkeit eine Infektionskrankheit zu erleiden. Das Ausmaß der Gefährdung wird durch das Infektionsrisiko (Exposition und Kontagionsindex), den Manifestationsindex und die Schwere der Erkrankung (z. B. Letalität) bestimmt. Es gibt Risikofaktoren, die die Wahrscheinlichkeit der Übertragung und der anschließenden Manifestation einer Infektionskrankheit erhöhen und es gibt andere Risikofaktoren, die die Wahrscheinlichkeit eher senken. Risiko erhöhend wirken z. B. eine hohe Anzahl von infizierten und symptomatischen Patienten in einem relevanten Bereich, eine lange Aufenthaltsdauer mit infizierten Patienten in einem umgrenzten Raum, ein geringer Luftwechsel, eine lange Dauer des Kontaktes zu einem infektiösen Patienten, eine hohe Virulenz des Erregers oder eine hohe Suszeptibilität (Empfänglichkeit) des Exponierten. Andererseits können eine große interindividuelle Distanz, eine hohe Effektivität von Schutzmaßnahmen (z. B. Atemschutz, Händehygiene) oder eine Immunität der Exponierten (z. B. Schutzimpfung) das Risiko der Manifestation einer Infektionserkrankung senken. Hinzu kommt die Möglichkeit eine Erkrankung zu therapieren, wodurch ggf. die Dauer der Ausscheidung eines Mikroorganismus begrenzt werden kann [67].

Die Ableitung von Schutzmaßnahmen sollte auf der Basis einer Risikoanalyse bzw. Gefahrenanalyse erfolgen und nachvollziehbar sein (siehe auch Empfehlung „Personelle und organisatorische Voraussetzungen zur Prävention nosokomialer Infektionen " [68]). Dabei erfolgt eine Bewertung von Risiken vor allem im Hinblick auf die angestrebten Schutzziele. Diese sollten sowohl für Patienten als auch für Personal immer die Vermeidung einer Infektion und/oder einer (schweren) Erkrankung sein. Hierbei sind Personalschutz und Patientenschutz gleichrangig $\mathrm{zu}$ bewerten. Auf die Risikobewertung folgt die Ableitung risikominimierender Maßnahmen (Präventionsmaßnahmen) auf der Basis der Faktoren der Risikoanalyse, der Eigenschaften des Erregers (bzw. des Erregerspektrums) und der Übertragungswege.

Basierend auf diesen Überlegungen kann die Risikoanalyse mit Ableitung der erforderlichen Maßnahmen auf 4 Faktoren aufgebaut werden (siehe $\bullet$ Tab. 1):

- Transmissionsweg

- Möglichkeit einer Impfung und besonderes Gefährdungs-/Transmissionspotential für Risikogruppen

- Räumliche Unterbringung

- Persönliche Schutzausrüstung

\subsection{Transmissionswege}

Es wird unterschieden zwischen einer Übertragung durch direkten oder indirekten Kontakt, durch Tröpfchen oder Aerosole sowie durch eine parenterale Exposition. Hinzu kommt bei einigen Erregern die an bestimmte Vektoren gebundene Transmission. Nicht zuletzt müssen ggf. auch über die Basishygiene hinausgehende Maßnahmen mit den für die Krankenhaushygiene Zuständigen (Hygieneteam) abgestimmt werden, wenn der Übertragungsweg eines Infektionserregers noch nicht genau bekannt ist.

Auch wenn in der Regel ein bestimmter Übertragungsweg der bedeutendste ist, kann in den meisten Fällen ein und derselbe Erreger auf verschiedene Weise übertragen werden (zum Beispiel durch Kontakt und durch Tröpfchen). Einige Erreger von Infektionskrankheiten sind nicht von Mensch zu Mensch übertragbar.

\subsubsection{Kontaktübertragung}

Kontaktübertragungen können in zwei wesentliche Untergruppen unterschieden 
werden: direkte und indirekte Übertragungsereignisse. Bei ersteren geschieht die Übertragung direkt von Person zu Person. Beispiele hierfür können sein:

a. die Übertragung von Ektoparasiten (z. B. Kopfläusen, Krätzmilben) von Kind zu Kind in entsprechenden Betreuungseinrichtungen;

b. die Übertragung von Shigellen oder Rotaviren von Patienten auf Klinikpersonal bei Kontakt mit den Exkreten (z. B. Stuhl) solcher Patienten. Früher wurde dieser Übertragungsweg als, fäkal-orale Übertragung bezeichnet. Indirekt ist diese z. B. auch durch die Kontamination von Lebensmitteln möglich.

Die indirekte Übertragung erfolgt unter Einbeziehung der Umwelt oder einer dritten Person. Hierbei ist die Bedeutung der möglichen Infektionskette Patient $A \rightarrow$ medizinisches Personal (Hände!) $\rightarrow$ Patient $B$ nicht zu unterschätzen. Ebenso können unbelebte Gegenstände (kontaminierte Oberflächen, Instrumente, etc.) diese Mittlerfunktion übernehmen. Insbesondere bei Erregern mit hoher Umweltpersistenz (Tenazität) stellt die Übertragung durch indirekten Kontakt ein ernstzunehmendes Problem dar [69]. Die meisten Erreger nosokomialer Infektionen können in der Umgebung des Patienten überleben und infektiös bleiben [31]. Beispiele hierfür sind Noro-, Rota- oder RS-Viren aber auch S. aureus, Acinetobacter baumannii und Clostridium difficile [31].

Auch die in Kliniken benutzte Berufskleidung (Arztkittel) sowie die Werkzeuge und Utensilien der täglichen Arbeit (Stethoskope, Thermometer, Blutdruckmanschetten etc.) können mit Erregern kontaminiert sein und zu Übertragungen im Sinne einer indirekten Kontakttransmission führen [70-72].

\subsubsection{Tröpfchenübertragung}

Streng genommen stellt der Übertragungsweg durch Tröpfchen eine distinkte Variante der Kontaktinfektion dar. Erreger, die über Tröpfchen übertragen werden, sind auch über direkte und indirekte Kontakte übertragbar. Der entscheidende Faktor für die Klassifizierung zur Tröpfcheninfektion ist die Übertragung durch ein Sekret/ Exkret aus dem Respirationstrakt, wel- ches direkt über einen kurzen Weg auf der Schleimhaut des Rezipienten auftrifft. Solche Sekrete entstehen z. B. beim Sprechen, Husten oder Niesen, aber auch bei medizinischen Interventionen (endotracheale Intubation, Absaugen aus den Atemwegen etc.). In der Regel beträgt die o. a. kurze Distanz ca. 1-2 m [57, 73-76], jedoch ist die Entfernung, die von Tröpfchen tatsächlich zurückgelegt wird, abhängig von patienteneigenen Faktoren wie Tröpfchendurchmesser und Ausstoßgeschwindigkeit, andererseits auch von diversen physikalischen Umgebungsvariablen wie Strömungsgeschwindigkeit und Turbulenzen, Luftdruck und -feuchtigkeit, Temperatur und kann unter entsprechenden Bedingungen bis zu $3 \mathrm{~m}$ betragen. Es muss berücksichtigt werden, dass Tröpfchen auch durch Beatmungsgeräte oder bei nicht-invasiver Beatmung austreten können, wenn die Ausatemluft bzw. die Abluft aus dem Beatmungsgerät nicht gefiltert ist [77-79].

Respiratorische Tröpfchen sind im Regelfall deutlich größer als $5 \mu \mathrm{m}$ im Durchmesser und gelangen daher im Unterschied zu Aerosolen (s. u.) nicht direkt mit der Atemluft in die terminalen Bronchioli bzw. in die Alveolen.

\subsubsection{Aerogene Übertragung}

Im Gegensatz zur Tröpfchenübertragung findet die aerogene Übertragung durch Tröpfchenkerne statt, deren Durchmesser weniger als $5 \mu \mathrm{m}$ beträgt. Die World Health Organization unterscheidet Partikelgrößen größer oder kleiner als $5 \mu \mathrm{m}$, um die Tröpfchenübertragung ( $>5 \mu \mathrm{m}$ ) von der aerogenen Übertragung durch Tröpfchenkerne ( $\leq 5 \mu \mathrm{m}$; Aerosole) abzugrenzen [80]. Aerosole $\leq 5 \mu \mathrm{m}$ gelangen mit der Atemluft in die tiefen Atemwege und umgehen damit wichtige physikalische und immunologische Barrieren.

Aufgrund ihrer geringen Größe sedimentieren Tröpfchenkerne wenn überhaupt nur sehr langsam und können damit in der Luft schwebend über größere Distanzen verbreitet werden. Eine Voraussetzung für aerogene Übertragung ist, dass die entsprechenden Mikroorganismen unter diesen Bedingungen über längere Zeit infektiös bleiben. Dann sind Übertragungen auch auf Personen möglich, die sich in unterschiedlichen Räumen befinden oder die sich zu unterschiedli- chen Zeiten im gleichen Raum aufhalten [81-83]. Beispiel für aerogen übertragbare Infektionen sind Masern, Varizellen und die offene Tuberkulose der Atemwege. Bei der Influenza sind die meisten Übertragungen durch Kontakt und durch Tröpfchen, jedoch nicht durch aerosol-gebundene Viren bedingt, obwohl ein solcher Übertragungsweg unter bestimmten Umgebungsbedingungen möglich ist [76].

\subsubsection{Parenterale Übertragung}

Bei der parenteralen Übertragung gelangt der Erreger über die nicht intakte Haut oder Schleimhaut bzw. durch Injektionen oder Punktionen, z. B. über Gefäßkatheter oder Verwendung von kontaminierten Spritzen in den Körper [2].

\subsubsection{Vektorassoziierte Übertragung}

Einige Infektionserkrankungen werden über sogenannte Vektoren übertragen. Es handelt sich hierbei um lebende Organismen, zumeist Insekten, die Mikroorganismen übertragen, jedoch nicht selbst erkranken. Diese Art der Übertragung spielt in medizinischen Einrichtungen in Deutschland nur eine untergeordnete Rolle.

\subsubsection{Unbekannter Übertragungsweg}

Bei neu auftretenden Krankheitserregern müssen - bis genaue Kenntnisse zu den Übertragungswegen vorhanden sind - ggf. maximale Maßnahmen zur Prävention der weiteren Verbreitung ergriffen werden. Durch Vergleich mit Erregern mit ähnlichem biologischen Verhalten und Krankheitsmanifestationen sollten entsprechende Hygienemaßnahmen eingeleitet werden, bis der Übertragungsweg genauer beschrieben ist. Ein Beispiel hierfür sind humane Bocaviren, die Atemwegsinfektionen auslösen und sich in Bezug auf ihre Tenazität wahrscheinlich ähnlich wie das humane Parvovirus B19 verhalten [84].

\subsection{Impfung und besonderes Gefährdungs- oder Transmissionspotential für Risikogruppen}

Eine aktive oder passive Immunisierung kann bei nicht immunen Menschen das 
Risiko einer Erkrankung durch bestimmte Erreger reduzieren. Bei der aktiven Immunisierung kann die Impfantwort durch eine Immundefizienz oder Immunsuppression des Geimpften beeinträchtigt sein. Bei hochgradiger Immunsuppression sind Impfungen mit Lebendimpfstoffen kontraindiziert [85]. Bei einigen Erkrankungen wird eine postexpositionelle Impfung empfohlen [86]. Von entscheidender Bedeutung zur Vermeidung nosokomialer Übertragungen bestimmter impfpräventabler Erkrankungen ist die Impfung des medizinischen Personals [86-89]. Diesen Überlegungen wird durch die Kategorie ,Impfung in $\bullet$ Tab. 1 Rechnung getragen. Berücksichtigt werden muss auch, dass bei bestimmten Erregern auch die Immunität (sei es durch eine Impfung oder eine durchgemachte Erkrankung) nicht vor einer vorübergehenden Besiedlung mit dem Erreger schützt (z. B. Pertussis).

\subsubsection{Impfpräventable Erkrankungen}

Das Risiko der Infektion und der Übertragung von Erkrankungen in Einrichtungen des Gesundheitssystems sowohl für die Beschäftigten als auch für die von ihnen betreuten Patienten kann durch Impfungen reduziert werden. Entsprechend werden von der Ständigen Impfkommission beim Robert Koch-Institut (STIKO) [86] Impfungen gegen Hepatitis A, Hepatitis B, Influenza, Masern, Mumps, Pertussis, Poliomyelitis, Röteln und Varizellen als Impfungen auf Grund eines erhöhten beruflichen Risikos empfohlen z. B. nach Gefährdungsbeurteilung gemäß Arbeitsschutzgesetz/Biostoffverordnung/Verordnung zur arbeitsmedizinischen Vorsorge (ArbMedVV) und dem G 42 (Berufsgenossenschaftlicher Grundsatz G 42 „Tätigkeiten mit Infektionsgefährdung"). Die Details der Empfehlungen können den jeweils aktuellen Publikationen der STIKO entnommen werden.

Bei bekannter Immunität kann nach TRBA 250 ggf. auf spezielle, über die Basishygiene hinausgehende Schutzmaßnahmen im Krankenhaus verzichtet werden. Der Impfstatus des Personals sollte den Beschäftigten selbst und dem Betriebsarzt bekannt sein. Im Zweifel über den Impfstatus sind entsprechen- de Schutzmaßnahmen anzuwenden [2]. Bevor auf Schutzmaßnahmen verzichtet wird, muss sichergestellt sein, dass eine entsprechende Immunität vorliegt. Details erläutern die Empfehlungen der STIKO.

\subsubsection{Besonderes Transmissionsrisiko für Immunsupprimierte}

Angeborene oder erworbene Immunsuppression kann zu besonderer Suszeptibilität für Infektionserkrankungen führen. Die Ausprägung des Defektes beeinflusst dabei den Verlauf der Infektionen für die eine besondere Empfindlichkeit besteht, z. B. virale Infektionen bei T-Zell-Defekten, bakterielle und invasive Pilzinfektionen bei Neutropenie oder Defekten der Granulozyten-/Makrophagenfunktion. Auch Patienten mit autoimmunologischen Erkrankungen, die außerhalb onkologischer Zentren behandelt werden, erhalten inzwischen zunehmend eine immunsuppressive Therapie, die sich unter Umständen langfristig auf die Suszeptibilität gegenüber bestimmten Infektionskrankheiten auswirkt. Spezielle Maßnahmen zur Infektionsprävention bei immunsupprimierten Patienten sind in den Empfehlungen der KRINKO „Anforderungen an die Hygiene bei der medizinischen Versorgung von immunsupprimierten Patienten " zusammengestellt [8]. Diese Maßnahmen können von Maßnahmen, die für Immunkompetente notwendig sind, abweichen.

Wichtig ist z. B., dass Patienten, die zu Beginn der intensiven Chemotherapie seropositiv sind, ihre Immunität unter der anhaltenden Immunsuppression verlieren können; bei diesen Patienten kann es zu Durchbruchsinfektionen z. B. durch Varizella-Zoster-Virus kommen [90, 91]. Außerdem können ,klassische Infektionskrankheiten' atypisch verlaufen, wie z. B. eine disseminierte Varizelleninfektion ohne Exanthem [92, 93].

\subsubsection{Besondere Risiken bei Transmission in der Schwangerschaft}

Bei Schwangeren besteht das Risiko eines komplizierten Verlaufs bestimmter Infektionen (z. B. Influenza). Hinzu kommt das Risiko einer konnatalen oder peripar- talen Infektion des Kindes. Daher sind für geburtshilfliche Abteilungen bzw. für schwangere, nicht-immune Beschäftigte besondere Schutzmaßnahmen zu ergreifen. So müssen schwangere Mitarbeiterinnen von der Behandlung kontagiöser Patienten (Art der Infektionen siehe - Tab. 1) ausgeschlossen werden [94, 95].

\subsection{Räumliche Unterbringung}

\subsubsection{Standardunterbringung}

Bei Erkrankungen, die nicht von Mensch zu Mensch, parenteral oder durch Vektoren übertragen werden, ist eine Unterbringung im Einzelzimmer nicht erforderlich. Auch bei kontagiösen Erkrankungen, zu deren Übertragung eine hohe Infektionsdosis erforderlich ist, kann auf die Unterbringung im Einzelzimmer verzichtet werden, wenn bei dem Patienten eine gute Compliance vorliegt, d. h. der Patient muss in der Lage sein, die empfohlenen Verhaltensweisen zu befolgen.

\subsubsection{Isolierzimmer}

Das Isolierzimmer ist ein zur Einzelunterbringung nutzbares Zimmer mit einem eigenen Bad und einer Toilette mit entsprechenden Händedesinfektionsmittelspendern und einem ausreichend groß dimensionierten Eingangsbereich, in dem Schutzkleidung so abgelegt und vor Verlassen des Zimmers entsorgt werden kann, dass es nicht zur Kontamination kommt. Der Patient darf dieses Zimmer nur nach Rücksprache mit dem Personal verlassen.

Ein Zusammenhang zwischen baulichen Gegebenheiten, z. B. der Zahl der Einzelzimmer und dem Auftreten von nosokomialen Infektionen, konnte bisher nicht eindeutig nachgewiesen werden [96-98]. Dennoch ist ein wichtiges Prinzip der Prävention der Erkrankungsübertragung Distanz zwischen infektiösen und empfänglichen Personen zu schaffen.

Eine Einzelunterbringung ist erforderlich, wenn die Übertragung des Erregers auf dem Luftweg/aerogen oder über respiratorische Sekrete (Tröpfcheninfektionen) erfolgen kann. Die Empfehlung zur Einzelunterbringung kann auch begründet sein in der Besonderheit einzelner Erkrankungen oder einzelner Erkrankungsphasen (profuse Durchfälle), bei schwer kontrollierbarem Übertragungsweg (z. B. 


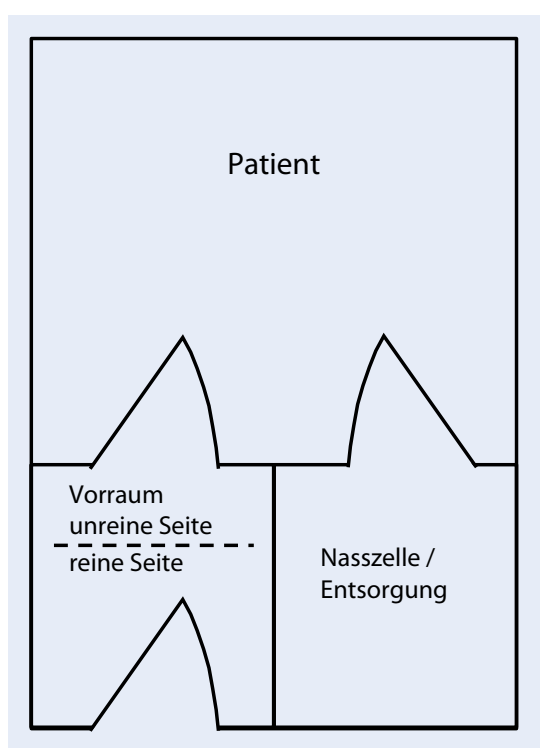

Abb. $1 \Delta$ Schematische Darstellung einer möglichen Anordnung von Vorraum und Nasszelle bzw. Entsorgungsraum

Ektoparasiten), oder bei Patienten, die nicht in der Lage sind, Maßnahmen der Basishygiene einzuhalten.

Eine gemeinsame Isolierung mehrerer Patienten (Kohortenisolierung) kann durchgeführt werden, wenn bei mehreren Patienten die gleichen Erreger nachgewiesen wurden und wenn nicht andere Gründe (z. B. Immunsuppression, Möglichkeit der Superinfektion oder Kolonisierung durch ein anderes Isolat oder einen anderen Stamm der gleichen oder einer verwandten Erregerspezies mit anderen Resistenz- oder Pathogenitätseigenschaften) dagegen sprechen.

Damit Patienten mit kontagiösen Erkrankungen ggf. in einem Einzelzimmer isoliert werden können, müssen als Einzelzimmer nutzbare Räumlichkeiten in angemessener Zahl verfügbar sein. Eine sehr häufige Rückmeldung aus der klinischen Praxis ist, dass die Zahl der verfügbaren Einzelzimmer nicht dem medizinisch begründeten Bedarf entspricht [99].

Aus der Perspektive der Infektionsprävention richtet sich der Bedarf an Einzelzimmern der jeweiligen Station nach dem Anteil der Patienten

- von denen ein erhöhtes Übertragungsrisiko ausgeht

- mit kontagiösen Erkrankungen, die eine Einzelzimmerisolierung erfordern
- die mit bestimmten multiresistenten Krankheitserregern besiedelt sind und daher in einem Einzelzimmer isoliert werden müssen [70]

- die protektiv (zu ihrem eigenen Schutz) isoliert werden müssen.

Beispielsweise wird in der Empfehlung zur Infektionsprävention bei Immunsupprimierten [8] darauf hingewiesen, dass mindestens $40 \%$ (besser 50\%) der Zimmer zur Isolierung (als Einzelzimmer nutzbares Zimmer mit eigenem Bad und Toilette mit entsprechenden Händedesinfektionsmittelspendern und einem ausreichend groß dimensionierten Eingangsbereich, in dem Kittel, Handschuhe und Mund-Nasen-Schutz angelegt und vor Verlassen des Zimmers entsorgt werden können) genutzt werden können.

Auch in den britischen National Health Service Empfehlungen wird für neu zu errichtende medizinische Einrichtungen ein Anteil von $50 \%$ Einzelzimmern gefordert [100].

Da bereits derzeit etwa 1,5 bis $4 \%$ der Patienten mit MRSA besiedelt sind [10] und der Anteil multiresistenter Erreger eher zunimmt, sollte allein deshalb bei Neuplanungen oder Sanierungen auf Normalpflegestationen ein Anteil von Einzelzimmerbetten an der Gesamtbettenzahl von 10-20\% nicht unterschritten werden.

Der genau erforderliche Anteil an Einzelzimmern in Krankhäusern sollte von der ärztlichen Leitung und der Krankenhausadministration in Zusammenarbeit mit dem zuständigen Krankenhaushygieniker (bzw. der Hygienekommission) abteilungsbezogen festgelegt und damit an den tatsächlichen Bedarf angepasst werden.

\subsubsection{Isolierzimmer mit Vorraum}

Ein Vorraum dient als Trennungszone zwischen dem kontaminierten Patientenzimmer und dem nicht kontaminierten Außenbereich. Um diese Funktion zu erfüllen, muss sie groß genug sein, um sie in eine reine und eine unreine Seite trennen zu können. Es ist nicht sinnvoll, Vorräume als schmalen Flurbereich zwischen Patientenzimmer und Nasszelle bzw. Patientenzimmer und Entsorgungsraum anzulegen. Die Nasszelle soll direkt vom Patientenzimmer aus zugänglich sein.
Eine mögliche Anordnung der Räume für künftige Bauvorhaben ist in • Abb. 1 schematisch dargestellt.

Eine spezielle Belüftung von Vorräumen ist in der Regel nicht erforderlich. Durch wechselseitiges Schließen der Türen des Vorraumes ist eine ausreichende Abgrenzung des Patientenzimmers vom Flur zu gewährleisten. Ist aus baulichen Gründen eine RLT-Anlage notwendig, z. B. aufgrund der erforderlichen Be- bzw. Entlüftung innenliegender Räume, so ist es empfehlenswert durch entsprechende Regelung der Abluft einen Unterdruck im Vorraum herzustellen. Patienten mit Masern, Windpocken oder multiresistenter Tuberkulose sollen bevorzugt in Isolierzimmern mit Vorräumen isoliert werden.

\subsubsection{Isolierstationen}

Einheiten/Stationen zur Isolierung infektiöser Patienten mit mehreren Isolierzimmern bieten sich im Allgemeinen nur in Krankenhäusern mit Fachdisziplinen an, die häufig kontagiöse Patienten stationär behandeln (z. B. Tuberkulose in der internistischen Pneumologie, virale Atemwegsinfektionen oder Gastroenteritiden in der Pädiatrie). Bei der Planung ist die geeignete Abtrennung der Zimmer untereinander und $\mathrm{zu}$ anderen Bereichen des Krankenhauses unter Vermeidung von Durchgangsverkehr zu berücksichtigen.

Neben den Isolierzimmern mit ausreichend großen Vorräumen und Sanitäreinrichtungen bevorzugt mit Steckbeckenspülen, müssen folgende Räumlichkeiten in ausreichender Größe auf der Station vorhanden sein:

\section{- Besucherraum}

- reiner Pflege-Arbeitsraum

- unreiner Pflege-Arbeitsraum/räume mit Steckbeckenspüle, sofern diese nicht den Zimmern zugeordnet sind

- Dienstraum oder - platz für Pflegekräfte

- Arztdienstraum

- Verteiler-, Stations- oder Teeküche

- Geräte- und Lagerräume

- Raum für die Bettenaufbereitung und/oder die Bereitstellung aufbereiteter Betten außerhalb der Dienstzeiten einer Bettenzentrale

- Entsorgungsraum, bevorzugt mit Zugang von außerhalb

- Personalaufenthaltsraum 
- Personalumkleideraum mit Schränken für Bereichskleidung oder Doppelschränken (Trennung von Dienstund Privatkleidung)

- Personaltoiletten

- Raum für Putzutensilien bzw. die Ausrüstung der Reinigungskräfte

Bei Planung von Untersuchungs- und Behandlungsräumen müssen Zugangswege, Ausstattung (leicht desinfizierbare Oberflächen) und ggf. Zugangsbeschränkungen besonders berücksichtigt werden.

Wenn in Isoliereinheiten Patienten verschiedener Fachdisziplinen zusammengefasst werden sollen, so ist zu gewährleisten, dass sich die Versorgung der Patienten dadurch nicht verschlechtert. Personal, das nur zeitweilig in diesem Bereich tätig ist, muss besonders geschult werden. Die Schulung ist zu dokumentieren [2].

\subsection{Persönliche Schutzausrüstung}

Das Tragen persönlicher Schutzausrüstung ist in vielen Fällen Bestandteil der Basishygiene, da Infektionen auch unerkannt vorliegen können.

Dabei wird die Schutzausrüstung in Abhängigkeit von der mit hoher Wahrscheinlichkeit zu erwartenden (vorhersehbaren) Exposition getragen. Liegt bei einem $\mathrm{Pa}$ tienten eine nachgewiesene Infektion vor oder ist eine Infektion hochwahrscheinlich, so wird die Schutzausrüstung bereits angelegt, wenn die Möglichkeit eines Kontaktes besteht. Dies kann bedeuten, dass die Ausrüstung bereits vor Zutritt zum Patientenzimmer angelegt wird.

\subsubsection{Schutzhandschuhe}

Bereits im Rahmen der Basishygiene wird empfohlen, nicht-sterile Einmalhandschuhe zu tragen, wenn direkter Kontakt mit Blut, Urin, Sekreten, Exkreten (Stuhl, Eiter), Schleimhäuten, nicht intakter Haut oder anderem potentiell infektiösem Material zu erwarten ist. Um einen ausreichenden Personalschutz beim Umgang mit Patienten, die an bestimmten Infektionserkrankungen erkrankt sind, zu gewährleisten, sollen Schutzhandschuhe (entsprechend DIN EN 420 (Schutzhandschuhe) und DIN EN 374 Teil 1) getragen werden [21, 22]. Die Handschuhe sollen bereits angelegt werden, wenn der
Kontakt auch nur möglich ist. Dies kann in der Praxis bedeuten, dass die Einmalhandschuhe bereits vor Betreten des Zimmers angelegt werden, insbesondere wenn eine erhebliche Kontamination der Oberflächen des Patientenzimmers zu erwarten ist. Nach Ablegen der Einmalhandschuhe ist stets eine Händedesinfektion erforderlich. Einmalhandschuhe werden somit nicht anstelle sondern zusätzlich zur Händedesinfektion eingesetzt [4].

\subsubsection{Schutzkittel}

Schutzkittel haben die Aufgabe zu verhindern, dass die Arbeitskleidung der Beschäftigten mit Mikroorganismen kontaminiert wird und dadurch die Beschäftigten direkt oder andere Patienten indirekt gefährdet werden. Geeignet sind langärmelige, mindestens flüssigkeitsabweisende Kittel mit Rückenschluss und Abschlussbündchen an den Armen (z. B. nach DIN EN 14126:2004-01), die entweder desinfizierbar sind oder als Einmalkittel entsorgt werden. Bei Durchfeuchtung ist der Kittel zu wechseln. Bei zu erwartender Durchfeuchtung sind ggf. Plastikschürzen anzulegen.

\subsubsection{Atemschutz}

In medizinischen Einrichtungen wird bei Atemschutz in erster Linie an den Mund-Nasen-Schutz (MNS) gedacht. Der Mund-Nasen-Schutz ist ein Medizinprodukt, das, vom Beschäftigten getragen, wirkungsvoll die Übertragung makroskopischer Tröpfchen aus dem Respirationstrakt von Patienten auf die Mundund Nasenschleimhaut des Trägers verhindern kann. Zudem werden Mund und Nase des Trägers vor Berührungen durch kontaminierte Hände geschützt [101]. Gemeinsam mit anderen Präventionsmaßnahmen (schnelle Diagnose, gezielte Therapie, Schutzkittel, Handschuhe und ggf. räumliche Isolierung) hat sich der MNS als wirksamer Bestandteil von Präventionsmaßnahmen bewährt [102]. Der MNS gehört jedoch nicht zum klassischen Atemschutz im Sinne des Arbeitsschutzes, weil er nicht über den erforderlichen Sitz und in der Regel nicht über das erforderliche Rückhaltevermögen gegenüber Aerosolen verfügt und deshalb nicht hinreichend vor dem Einatmen von Aerosolen schützen kann.
Im Gegensatz zum Mund-NasenSchutz (MNS), der bestimmungsgemäß die Umgebung vor der Nasen-Rachenflora des Trägers schützen soll, hat der Atemschutz die Aufgabe, den Träger selbst vor Schadstoffen aus der Umgebung zu schützen, welche durch die Luft übertragen werden (Gase und Mikroorganismen).

Der Atemschutz ist somit eine Maßnahme des Arbeitsschutzes. Als geeignet werden in medizinischen Einrichtungen partikelfiltrierende Atemschutzmasken, sog. Filtering Face Pieces -FFP angesehen, die eine Reduktion infektiöser Aerosole in der eingeatmeten Luft bewirken. Nach DIN EN 149 wird die Gesamtleckage dieser Atemschutzmasken geprüft, die sich aus Filterdurchlass und der sogenannten Verpassungsleckage (Undichtigkeiten zwischen der Dichtlinie der Maske und dem Gesicht des Trägers) zusammensetzt. Entsprechend der sich daraus ergebenden Gesamtleckage werden die Filtermasken in drei Klassen unterteilt, mit jeweils einer zulässigen Leckage von:

- FFP1 max. $22 \%$

- FFP2 max. $8 \%$

- FFP3 max. $2 \%$.

Entscheidend für die Wirksamkeit der FFP-Maske ist neben den Filtereigenschaften vor allem der Dichtsitz (Passform), weshalb die angegebenen $\mathrm{Re}$ duktionswerte nur bei optimal angelegter Maske erreicht werden können. Barthaare im Bereich der Dichtlinie zwischen Atemschutzmaske und Gesichtshaut können die Schutzwirkung der Schutzmaske beeinträchtigen. Darauf sollten Bartträger hingewiesen werden. In der Regel stellt das Tragen einer gut angepassten FFP2Maske aber einen geeigneten Schutz vor infektiösen Aerosolen, einschließlich Viren dar, da davon ausgegangen werden kann, dass diese an kleinste Tröpfchen oder Tröpfchenkerne gebunden sind [2].

Ab FFP 2 kann je nach Tätigkeit eine arbeitsmedizinische Vorsorgeuntersuchung nach ArbMedVV (G26) in Verbindung mit der Arbeitsmedizinischen Regel AMR 14.2 „Einteilung von Atemschutzgeräten in Gruppen “ erforderlich sein [103]. Auf jeden Fall ist die Einbeziehung des Betriebsarztes und eine Gefährdungsbeurteilung nach Arbeitsschutzgesetz zu empfehlen [104]. Die arbeitsmedizinische 
Problematik lässt sich durch die Verwendung von FFP-Masken mit Ausatemventil entschärfen, wobei darauf zu achten ist, dass dadurch keine Gefährdung der Patienten entsteht.

In der TRBA 250 wird für die Praxis konkretisiert, dass bei Tätigkeiten an oder in unmittelbarer Nähe von Patienten, die an luftübertragbaren Krankheitserregern erkrankt sind, mindestens FFP2Masken getragen werden sollen. Dies gilt insbesondere, wenn die Beschäftigten dabei Hustenstößen der Patienten ausgesetzt sein können. Auf das Tragen der FFP2Masken durch den Beschäftigten kann im Einzelfall, in Abhängigkeit von der Erkrankung des Patienten, verzichtet werden, wenn bekannt ist, dass der betroffene Beschäftigte über einen ausreichenden Immunschutz, z. B. aufgrund einer Impfung, verfügt [2].

Werden Tätigkeiten an Patienten, die an luftübertragbaren Krankheiten erkrankt sind, ausgeführt, und trägt der Patient einen MNS, reicht nach der TRBA 250 für den Behandler das gleichzeitige Tragen eines MNS als geeignete Präventionsmaßnahme in der Regel aus. Dies gilt nicht, wenn der Erreger der Risikogruppe 3 oder gar 4 zugeordnet ist [105].

Das Ausmaß des präventiven Werts des Atemschutzes kann nur schwer abgeschätzt werden, da entsprechende gut geplante und durchgeführte Studien fehlen. Metaanalysen verschiedener retrospektiver Untersuchungen zeigen jedoch eine signifikante Reduktion der Verbreitung respiratorischer Erkrankungen, wenn ein Atemschutz in die Präventionsmaßnahmen einbezogen war [106-111].

Ein Review zur Infektionsübertragung durch Aerosol-generierende medizinische Maßnahmen zeigte, dass das höchste Risiko einer Übertragung bei trachealer Intubation, nicht-invasiver Beatmung, Tracheotomie und der Beatmung mit Beatmungsbeutel und Maske vor der Intubation entsteht [79].

Daher soll bei durch Tröpfchen oder aerogen übertragenen Erkrankungen das Personal einen Mund-Nase-Schutz oder einen Atemschutz tragen, der der Art des infektiösen Materials (Tröpfchen oder Tröpfchenkerne) und den Umständen der Aerosolentstehung angepasst wird (arbeitsplatzspezifische Gefährdungsanalyse).

\subsubsection{Schutzbrille}

Bei einigen Erkrankungen, deren Erreger über die Konjunktiven übertragen werden kann, ist es erforderlich den Atemschutz mit einer Schutzbrille zu kombinieren, um eine Infektion über die Konjunktiven zu vermeiden [108].

\subsection{Umgang mit Medizinprodukten, Wäsche, Geschirr und Abfall}

In der Regel sind Maßnahmen der Basishygiene ausreichend, Infektionsübertragungen durch Medizinprodukte, Wäsche, Geschirr oder Abfall zu verhindern. In einigen wenigen Fällen gelten grundsätzlich andere Überlegungen (für Abfall siehe Vollzugshilfe zur Entsorgung von Abfällen aus Einrichtungen des Gesundheitsdienstes der Bund/Länder-Arbeitsgemeinschaft (LAGA) [58].

Der Umgang mit kontaminierten Materialien außerhalb des Isolierbereiches soll auf ein Minimum reduziert werden. Eine Reihe von Medizinprodukten kann als Einmalprodukte verwendet werden. Produkte, die einer Wiederverwendung zugeführt werden sollen, müssen vor Verlassen des Isolierbereiches mit geeigneten Mitteln desinfiziert oder in geeigneten fest verschlossenen und bei Verlassen des Isolierbereiches außenseitig desinfizierten und gekennzeichneten Behältern transportiert werden.

\subsection{Laufende \\ Desinfektionsmaßnahmen}

In Abhängigkeit von der Art des Infektionserregers kann es notwendig sein, laufende Desinfektionsverfahren anzupassen, insbesondere bei unbehüllten Viren oder Bakteriensporen.

\subsubsection{Händehygiene}

Werden Infektionskrankheiten durch bakterielle Sporen übertragen, so sind zur Prävention der Verbreitung für Kontakte mit potentiell infektiösem Material oder kontaminierten Flächen nicht-sterile Einmalhandschuhe zu tragen. Nach dem Ausziehen der Einmalhandschuhe erfolgt zusätzlich zur Händedesinfektion ein gründliches Waschen der Hände mit Seife.
Die üblicherweise eingesetzten Händedesinfektionsmittel sind begrenzt viruzid, so dass eine Wirksamkeit gegenüber unbehüllten Viren nicht generell gegeben ist. Daher muss bei durch unbehüllte Viren übertragbaren Erkrankungen auf entsprechend wirksame Händedesinfektionsmittel umgestellt werden.

\subsubsection{Flächendesinfektion}

Für die laufende Desinfektion sind die zur prophylaktischen Desinfektion verwendeten Desinfektionsmittelkonzentrationen und Einwirkzeiten ausreichend.

Für die Schlussdesinfektion (siehe Kap. 3.8) müssen die Konzentrationen und Einwirkzeiten des Desinfektionsmittels in Ihrer Wirksamkeit ggf. dem spezifischen Erreger der Erkrankung des isolierten Patienten angepasst werden. Ziel ist es den nachfolgenden Patienten, der in das Patientenzimmer verlegt wird, sicher vor der Infektion des vor ihm im Zimmer befindlichen Patienten zu schützen. Die notwendigen Konzentrationen zur Erreichung einer ausreichenden Viruzidie bzw. Sporozidie können so hoch sein, dass diese Konzentrationen für einen im Zimmer liegenden Patienten nicht tolerabel bzw. akut toxisch wären.

In jedem Fall (laufende und Schlussdesinfektion) ist durch korrekte Aufbereitung der Reinigungsutensilien direkt nach Gebrauch sicherzustellen, dass Mikroorganismen nicht verbreitet werden.

Die Aufbereitung von Gegenständen oder Geräten die im Isolierbereich genutzt wurden, muss mit Desinfektionsmitteln mit geeigneter Wirksamkeit erfolgen, z. B. viruzide oder sporizide Wirksamkeit.

\subsection{Transport des Patienten}

Patienten mit Nachweis bestimmter Erreger, aufgrund deren Erkrankung spezielle Schutzmaßnahmen notwendig sind, sollen nur transportiert oder verlegt werden, wenn dies aus medizinischen Gründen indiziert ist.

Innerhalb des Hauses müssen bei Verlegung oder auch Transport in apparativ-diagnostische Abteilungen (Röntgen, Computertomographie, Magnetresonanztomographie usw.) die notwendigen Informationen und Utensilien zu den erforderlichen Schutzmaßnahmen vor An- 
kunft des Patienten vorliegen. Bei Verlegung in andere Krankenhäuser, nachsorgende Einrichtungen oder Heime muss die Information über die infektiöse Erkrankung und ggf. über erforderliche Schutzmaßnahmen ebenfalls vorab weitergegeben werden.

Hat der Patient eine Erkrankung, die durch Tröpfchen oder Aerosole übertragen wird, soll er für den Transport - wenn möglich/zumutbar - einen Mund-NasenSchutz tragen.

Transporte sollten möglichst auf kürzestem Wege erfolgen, wobei jedoch Bereiche mit erhöhtem Patienten- oder Besucherverkehr gemieden werden sollen.

\subsection{Dauer und Beendigung der Maßnahmen}

\subsubsection{Dauer der Maßnahmen}

Die Schutzmaßnahmen müssen so lange aufrechterhalten werden, bis die Kontagiosität des Patienten so weit abgenommen hat, dass Personal und Mitpatienten nicht mehr gefährdet sind. Bis vor wenigen Jahren wurden die in $\bullet$ Tab. 1 aufgeführten Schutzmaßnahmen für die meisten Krankheiten über die Dauer der Symptomatik des Patienten aufrechterhalten. Zunehmend sensitivere Methoden des Erregernachweises haben gezeigt, dass einige Erreger zum Teil noch für längere Zeit nach Abklingen der Symptome ausgeschieden werden können [112-114]. Dies hängt auch von patientenspezifischen Faktoren ab (z. B. verlängerte Ausscheidung von Respiratory Syncytial Virus bei Früh- und Neugeborenen oder bei immunsupprimierten Patienten [115]).

Zusätzlich können bei bestimmten Erregern (z. B. Norovirus, EHEC) oder bei immunsupprimierten Patienten sehr kleine Erregermengen für eine Übertragung ausreichen, daher kann es erforderlich sein, Schutzmaßnahmen auch über die Dauer der Symptomatik des Patienten hinaus aufrecht zu erhalten.

In - Tab. 1 sind die Zeiten angegeben, nach denen davon auszugehen ist, dass für immunkompetente Kontaktpersonen keine erhöhte Infektionsgefahr mehr besteht. Im individuellen Fall oder für besondere Patientengruppen (z. B. in der Pädiatrie, bei hochgradig immunsupprimierten $\mathrm{Pa}$ tienten) kann dies bedeuten, dass auch bei bereits wieder asymptomatischen Patienten, die aus anderen Gründen weiter stationär behandelt werden müssen, Kontrollen der Erregerausscheidung erforderlich sind.

\subsubsection{Maßnahmen nach Beendigung der Isolierung}

Können die Isolierungsmaßnahmen beendet werden, so muss die Patientenumgebung soweit aufbereitet werden, dass von ihr für eine nicht-infizierte bzw. -kolonisierte Person kein erhöhtes Infektionsrisiko ausgeht. Eine zu diesem Zweck durchzuführende Schlussdesinfektion erstreckt sich je nach Erkrankung oder Krankheitserreger auf die patientennahen bzw. alle erreichbaren Oberflächen und Gegenstände einschließlich des Patientenbettes und der Nasszelle, die mit den Krankheitserregern kontaminiert sein können. In besonderen Fällen können andere Wirkstoffe oder Konzentrations-Zeit-Relationen und Verfahren als bei der routinemäßigen Desinfektion notwendig sein. Hierbei ist zu beachten, dass - das eingesetzte Desinfektionsmittel gegenüber dem Erreger wirksam sein muss [5]

- vom Reinigungspersonal geeignete Schutzmaßnahmen eingehalten werden.

Zur sicheren Abtötung bestimmter Erreger (z. B. bakterieller Sporen oder unbehüllter Viren) müssen entsprechend wirksame Desinfektionsmittel eingesetzt und die volle Einwirkzeit des Desinfektionsmittels abgewartet werden, bevor die Flächen wieder in Betrieb genommen werden können. Mit dem Krankenhaushygieniker ist nach Risikoanalyse festzulegen, welche Flächen und Gegenstände in die Schlussdesinfektion einzubeziehen sind.

\section{Umsetzung in der medizinischen Einrichtung}

Je höher die Compliance des medizinischen oder betreuenden Personals mit der Basishygiene ist, desto geringer ist die Wahrscheinlichkeit einer Gefährdung für Personal und Mitpatienten oder Mitbewohner durch Infektionserkrankungen. Daher steht die Umsetzung der Basishygiene im Vordergrund. Infektionserkran- kungen, die ggf. auch eine Gefährdung für das Personal darstellen, sind jedoch in der Regel Sondersituationen, in denen systematisch geplantes Handeln erforderlich ist. Schritte zur Einführung und Umsetzung der erforderlichen Hygienemaßnahmen sind im Hygieneplan festzulegen.

Hierzu gehören:

- Umsetzung der Meldepflicht (siehe Infektionsschutzgesetz und Länderverordnungen)

- Festlegung der umzusetzenden Maßnahmen unter Berücksichtigung des Schutzes für Personal und Mitpatienten bzw. Mitbewohner

- Einweisung von Patienten und ihren Angehörigen, Begleitpersonen oder Besuchern über die Händehygiene und weitere patientenbezogene Schutzmaßnahmen.

- Schulung des Personals zu Isolierungsmaßnahmen, Schlussdesinfektion, An- und Ablegen von Schutzkleidung

- Informationsweitergabe innerhalb und außerhalb der Einrichtung

Die festgelegten Maßnahmen müssen schnellstmöglich, sobald der begründete Verdacht auf eine Infektionserkrankung vorliegt, eingeleitet werden. Dies muss ggf. schon in der Notaufnahme erfolgen, so dass es sich anbietet, dort geeignete Räume zur Isolierung infektionsverdächtiger Patienten vorzuhalten. Kommen differentialdiagnostisch verschiedene Infektionserkrankungen in Betracht, so richten sich die Maßnahmen nach der Erkrankung mit dem Übertragungsweg, der die weitreichendsten Schutzmaßnahmen erfordert.

Die Empfehlungen wurden ehrenamtlich und ohne Einflussnahme kommerzieller Interessengruppen im Auftrag der Kommission für Krankenhaushygiene und Infektionsprävention bearbeitet von Frau Prof. Dr. Constanze Wendt (Leiterin der Arbeitsgruppe), Herrn Prof. Dr. Martin Exner, Herrn Dr. Thomas Grünewald, Herrn PD Dr. Heinz-Michael Just, Herrn Prof. Dr. Bernhard Ruf, Herrn Prof. Dr. Dr. Arne Simon, Frau Anna Triphaus, Herrn Prof. Dr. Martin Mielke. 
Tab. 1 Übersicht der Infektionserkrankungen und erforderliche Maßnahmen als Grundlage für Festlegungen im Hygieneplan

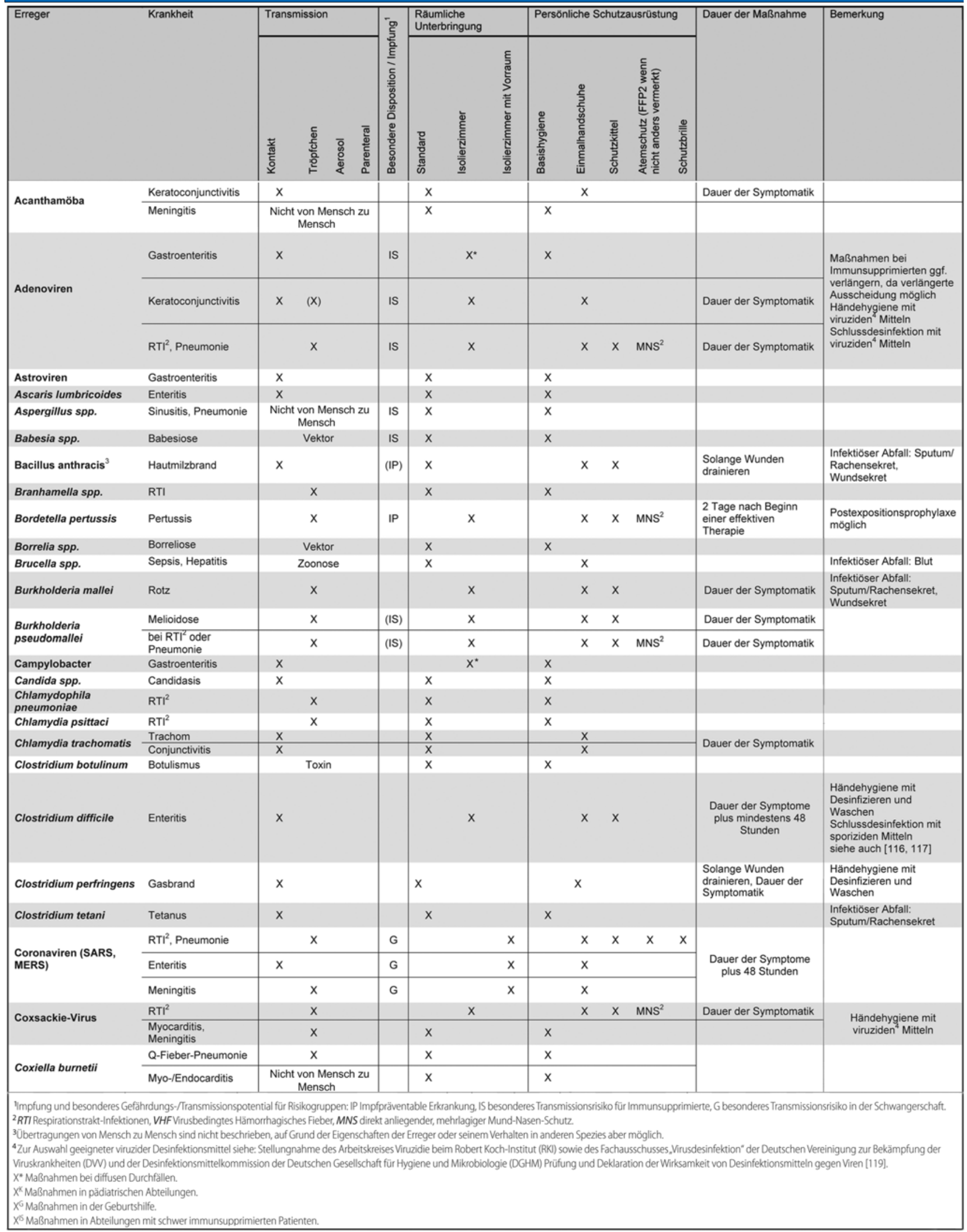


Tab. 1 Übersicht der Infektionserkrankungen und erforderliche Maßnahmen als Grundlage für Festlegungen im Hygieneplan (Fortsetzung)

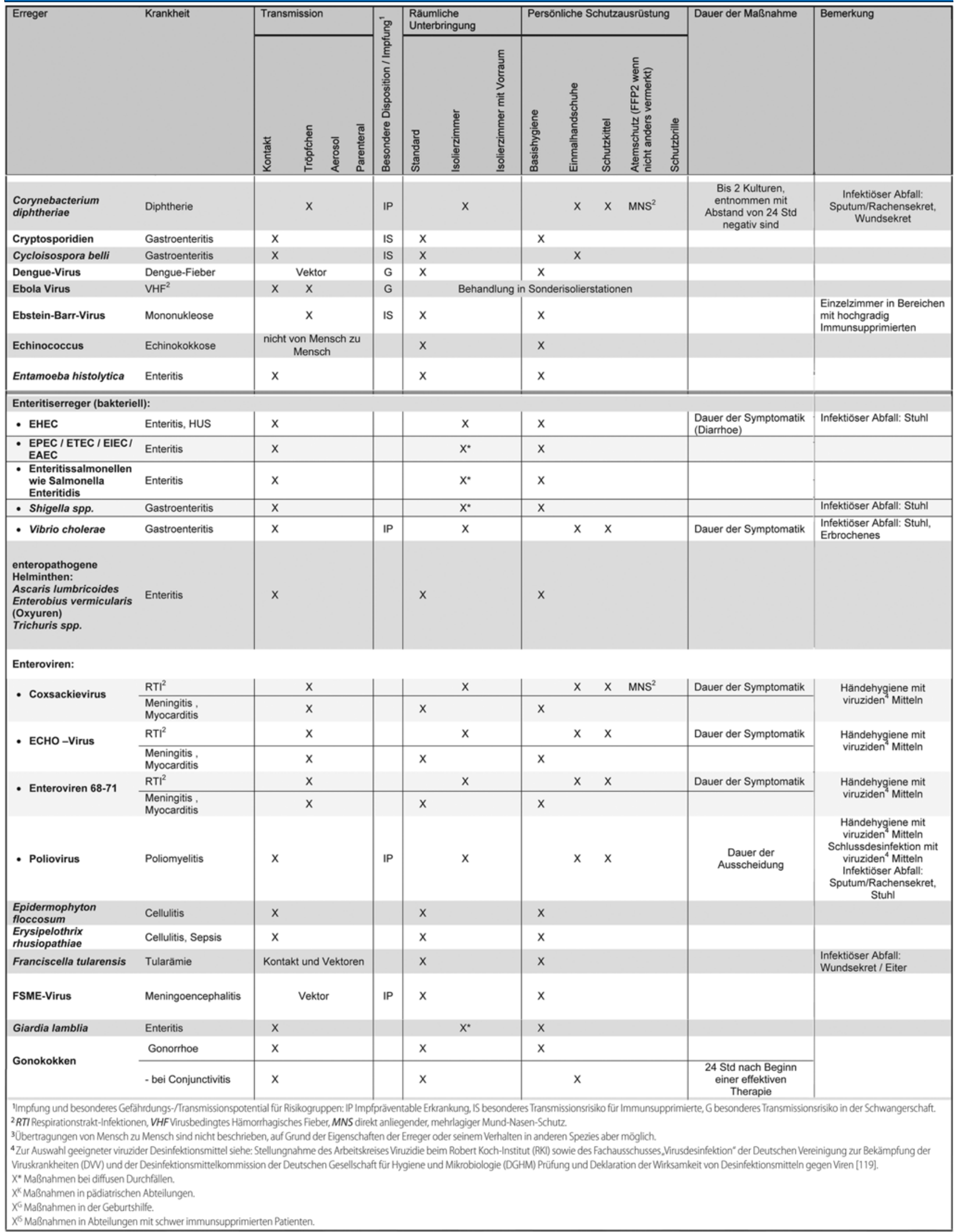


Tab. 1 Übersicht der Infektionserkrankungen und erforderliche Maßnahmen als Grundlage für Festlegungen im Hygieneplan (Fortsetzung)

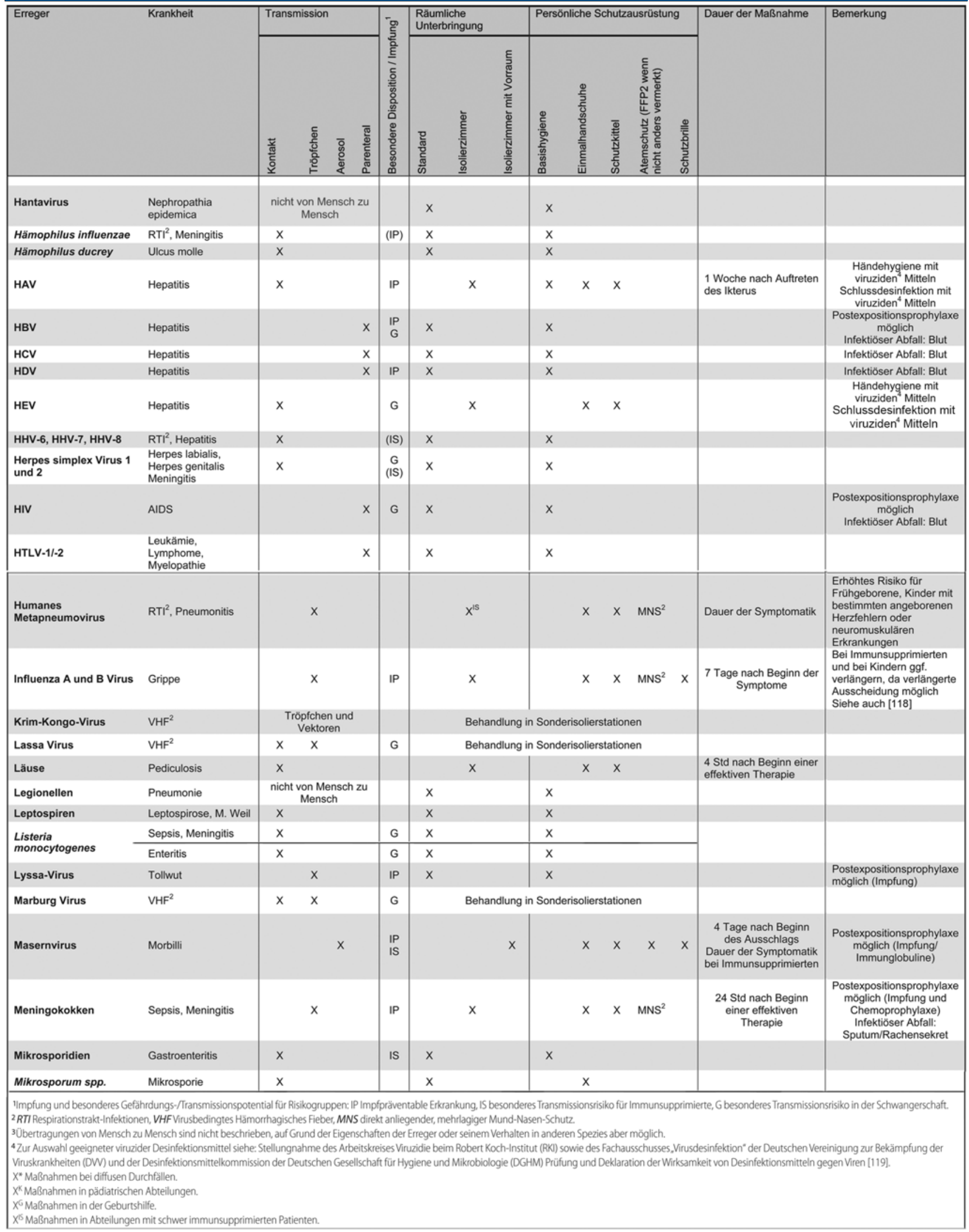


Tab. 1 Übersicht der Infektionserkrankungen und erforderliche Maßnahmen als Grundlage für Festlegungen im Hygieneplan (Fortsetzung)

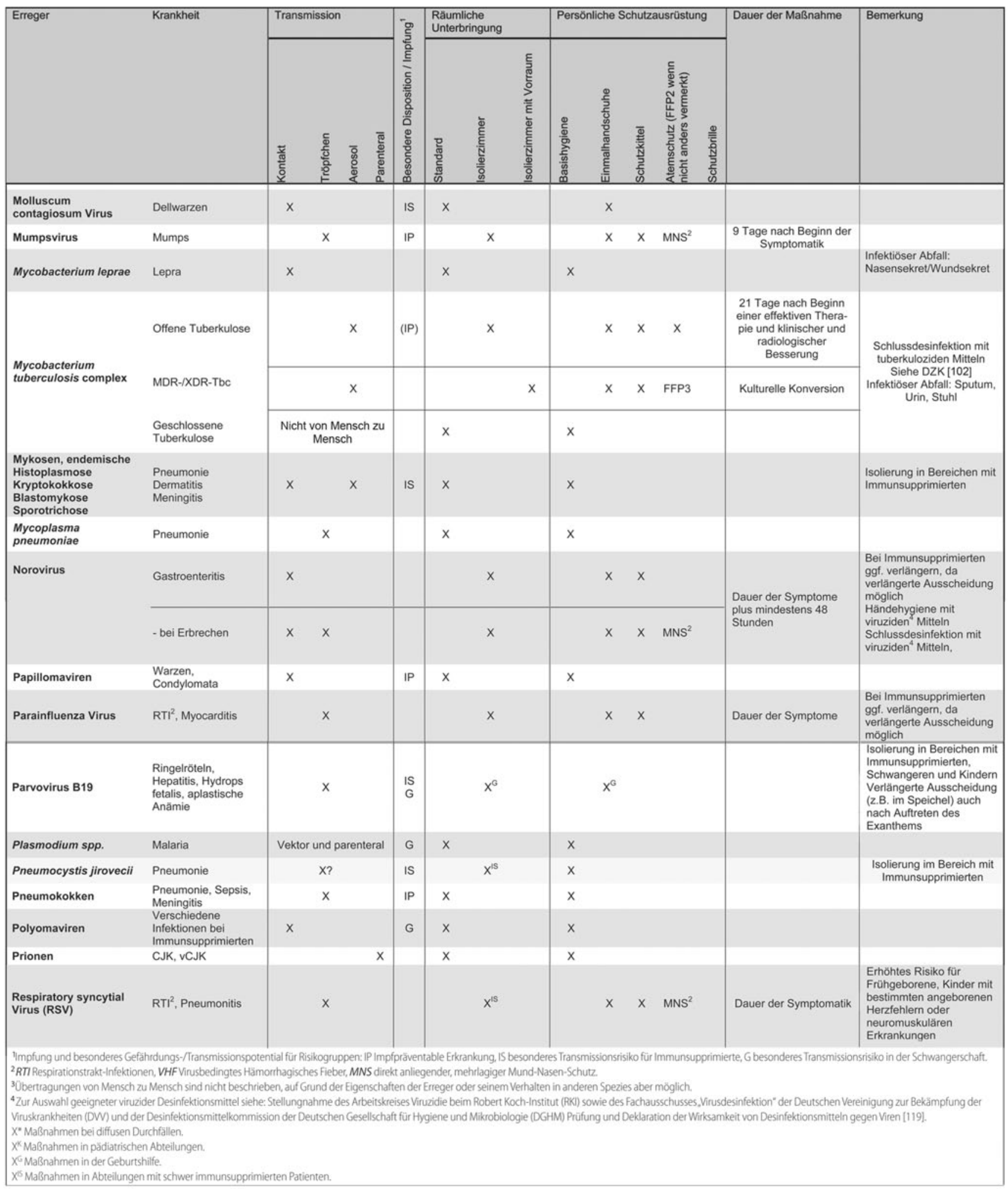


Tab. 1 Übersicht der Infektionserkrankungen und erforderliche Maßnahmen als Grundlage für Festlegungen im Hygieneplan (Fortsetzung)

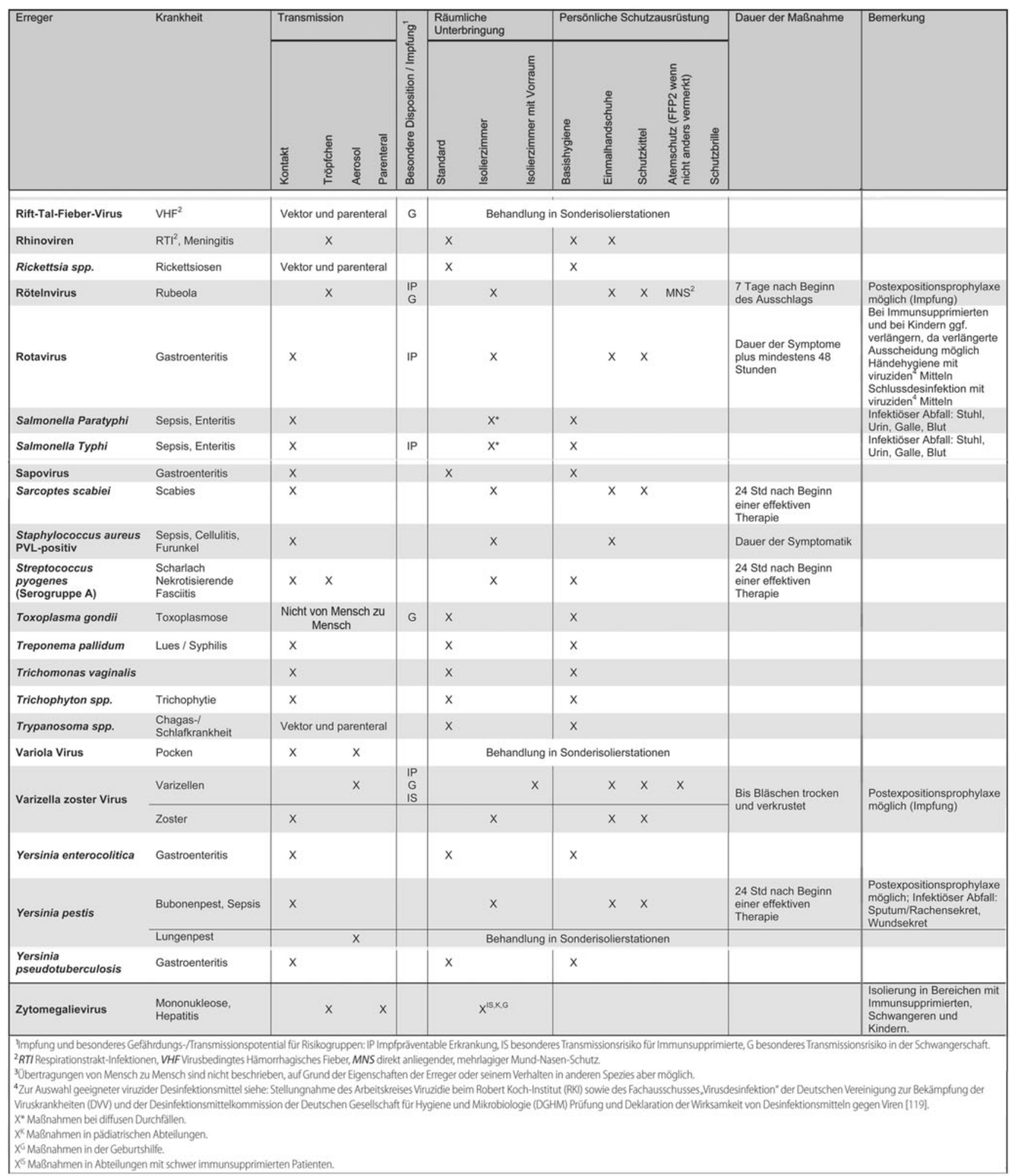




\section{Literatur}

1. Kiehl W, Robert Koch-Institut (Hrsg.) (2015) Infektionsschutz und Infektionsepidemiologie. Fachwörter - Definitionen - Interpretationen. Robert Koch-Institut: Berlin, URL: https://www.rki. de/DE/Content/Service/Publikationen/Fachwoerterbuch_Infektionsschutz.pdf;jsessionid $=20903$ E13C94B2D778F863BCE67CA7439.2 cid381?_blob=publicationFile

2. TRBA 250 - Biologische Arbeitsstoffe im Gesundheitswesen und in der Wohlfahrtspflege. GMBI 2014(10-11) Letzte Änderung vom: 21.07.2015

3. Grünewald TS (2015) Strukturelle Erfordernisse für das Management von Patienten mit hochkontagiösen, lebensbedrohlichen Erkrankungen - Update 2015. Bundesgesundheitsblatt 58:662670

4. Kommission für Krankenhaushygiene und Infektionsprävention (2000) Empfehlung zur Händehygiene. Bundesgesundheitsblatt Gesundheitsforschung Gesundheitsschutz 43:230-233

5. Kommission für Krankenhaushygiene und Infektionsprävention (2004) Empfehlung zu den Anforderungen an die Hygiene bei der Reinigung und Desinfektion von Flächen. Bundesgesundheitsblatt Gesundheitsforschung Gesundheitsschutz 47:51-61

6. Kommission für Krankenhaushygiene und Infektionsprävention (2012) Empfehlung zu den Anforderungen an die Hygiene bei der Aufbereitung von Medizinprodukten. Bundesgesundheitsbl 55:1244-1310

7. Kommission für Krankenhaushygiene und Infektionsprävention (2011) Empfehlung zu den Anforderungen an die Hygiene bei Punktionen und Injektionen. Bundesgesundheitsbl 54:1135-1144

8. Kommission für Krankenhaushygiene und Infektionsprävention (2010) Empfehlungen zu den Anforderungen an die Hygiene bei der medizinischen Versorgung von immunsupprimierten Patienten. Bundesgesundheitsbl 53:357-388

9. Kommission für Krankenhaushygiene und Infektionsprävention (2007) Empfehlung zur Prävention nosokomialer Infektionen bei neonatologischen Intensivpflegepatienten mit einem Geburtsgewicht unter $1500 \mathrm{~g}$. Bundesgesundheitsblatt Gesundheitsforschung Gesundheitsschutz 50:1265-1303

10. Kommission für Krankenhaushygiene und Infektionsprävention (2014) Empfehlungen zur Prävention und Kontrolle von Methicillin-resistenten Staphylococcusaureus-Stämmen (MRSA) in medizinischen und pflegerischen Einrichtungen. Empfehlung der Kommission für Krankenhaushygiene und Infektionsprävention (KRINKO) beim Robert Koch-Institut. Bundesgesundheitsblatt Gesundheitsforschung Gesundheitsschutz 57:696-732

11. Kommission für Krankenhaushygiene und Infektionsprävention (2012) Empfehlung zu Hygienemaßnahmen bei Infektionen oder Besiedlung mit multiresistenten gramnegativen Stäbchen. Bundesgesundheitsbl 55:1311-1354

12. Statistisches Bundesamt (DESTATIS) (2013) 18,6 Millionen Patienten 2012 stationär im Krankenhaus behandelt Pressemitteilung vom 28.08.2013, URL: https://www.destatis.de/ DE/PresseService/Presse/Pressemitteilungen/2013/08/PD13_286_231.html. Zugegriffen: 23. Juli 2015

13. Boyce J (2007) Environmental contamination makes an important contribution to hospital infection. J Hosp Infect 65:50-54
14. Dancer SJ (2009) The role of environmental cleaning in the control of hospital-acquired infection. J Hosp Infect 73:378-385

15. Donskey C (2013) Does improving surface cleaning and disinfection reduce health care-associated infections? Am J Infect Control 41:12-19

16. Otter JA (2011) The Role Played by Contaminated Surfaces in the Transmission of Nosocomial Pathogens. Infect Control Hosp Epidemiol 32(7):687-699

17. Otter JA (2013) Evidence that contaminated surfaces contribute to the transmission of hospital pathogens and an overview of strategies to address contaminated surfaces in hospital settings. Am J Infect Control 41:6-11

18. Weber DJ (2010) Role of hospital surfaces in the transmission of emerging health care-associated pathogens: Norovirus, Clostridium difficile, and Acinetobacter species. Am J Infect Control 38:2533

19. Kommission für Krankenhaushygiene und Infektionsprävention (KRINKO) (2007) Anforderungen der Krankenhaushygiene und des Arbeitsschutzes an die Hygienebekleidung und persönliche Schutzausrüstung. Epidemiol Bulletin 1:3-4

20. DIN EN 455:2015-07 Medizinische Handschuhe zum einmaligen Gebrauch. Beuth Verlag: Berlin

21. DIN EN 420:2010-03 Schutzhandschuhe - Allgemeine Anforderungen und Prüfverfahren. Beuth Verlag: Berlin

22. DIN EN 374-1:2003-12 Schutzhandschuhe gegen Chemikalien und Mikroorganismen - Teil 1:Terminologie und Leistungsanforderungen. Beuth Verlag: Berlin

23. Pittet $D$ (1999) Bacterial Contamination of the Hands of Hospital Staff During Routine Patient Care. Arch Intern Med 159(8):821-826

24. Tenorio AR (2001) Effectiveness of gloves in the prevention of hand carriage of vancomycin-resistant enterococcus species by health care workers after patient care. Clin Infect Dis 32(5):8269

25. Yin J, Schweizer ML, Herwaldt LA, Pottinger JM, Perencevich EN (2013) Benefits of universal gloving on hospital-acquired infections in acute care pediatric units. Pediatrics 131(5):1515-1520

26. Bearman G, Marra AR, Sessler CN, Smith WR, Rosato A, Laplante JK, Wenzel RP, Edmond MB (2007) A controlled trial of universal gloving versus contact precautions for preventing the transmission of multidrug-resistant organisms. Am J Infect Control 35(10):650-655

27. Bearman G., Rosato AE, Duane TM et al (2010) Trial of universal gloving with emollient-impregnated gloves to promote skin health and prevent the transmission of multidrug-resistant organisms in a surgical intensive care unit. Infect Control Hosp Epidemiol 31:491-497

28. Huskins W, Huckabee CM, O'Grady NP et al (2011) Intervention to reduce transmission of resistant bacteria in intensive care. N Engl J Med 364:1407-1418

29. Neely A, Maley MP (2001) Dealing with contaminated computer keyboards and microbial survival. Am J Infect Control 29:131-132

30. Neely A, Sittig DF (2002) Basic microbiologic and infection control information to reduce the potential transmission of pathogens to patients via computer hardware. J Am Med Inform Assoc 9:500-508

31. Kramer A, Schwebke I, Kampf G (2006) How long do nosocomial pathogens persist on inanimate surfaces? A systematic review. BMC Infect Dis 6:130
32. Wiener-Well Y, Galuty M, Rudensky B et al (2011) Nursing and physician attire as possible source of nosocomial infections. Am J Infect Control 39:555-559

33. Wilson J, Loveday HP, Hoffman PN, Pratt RJ (2007) Uniform: an evidence review of the microbiological significance of uniforms and uniform policy in the prevention and control of healthcare-associated infections. Report to the Department of Health (England). J Hosp Infect 66:301-307

34. Banu A, Anand M, Nagi N (2012) White coats as a vehicle for bacterial dissemination. J Clin Diagn Res 6(8):1381-1384

35. Burden M et al (2011) Newly cleaned physician uniforms and infrequently washed white coats have similar rates of bacterial contamination after an 8-hour workday: a randomized controlled trial. J Hosp Med 6(4):177-182

36. Huntley DE, Campbell J (1998) Bacterial contamination of scrub jackets during dental hygiene procedures. J Dent Hyg 72(3):19-23

37. Morgan D, Liang SY, Smith CL et al (2010) Frequent multidrug-resistant Acinetobacter baumannii contamination of gloves, gowns, and hands of healthcare workers. Infect Control Hosp Epidemiol 31(7):716-721

38. Snyder G, Thom KA, Furuno JP et al (2008) Detection of methicillin-resistant Staphylococcus aureus and vancomycin-resistant enterococci on the gowns and gloves of healthcare workers. Infect Control Hosp Epidemiol 29(7):583-589

39. Zachary K, Bayne PS, Morrison VJ et al (2012) Contamination of gowns, gloves, and stethoscopes with vancomycin-resistant enterococci. Infect Control Hosp Epidemiol 22(9):560-564

40. Treakle A, Thom KA, Furuno JP et al (2009) Bacterial contamination of health care workers white coats. Am J Infect Control 37(2):101-105

41. Bandi S, Conway A (2012) Question 2 does regular cleaning of stethoscopes result in a reduction in nosocomial infections? Arch Dis Child 97(2):175-177

42. Mehta A, Halvosa JS, Gould CV, Steinberg JP (2010) Efficacy of alcohol-based hand rubs in the disinfection of stethoscopes. Infect Control Hosp Epidemiol 31(8):870-872

43. Noskin G, Stosor V, Cooper I, Peterson LR (1995) Recovery of vancomycin-resistant enterococci on fingertips and environmental surfaces. Infect Control Hosp Epidemiol 16:577-581

44. Nunez S, Moreno A, Green K, Villar J (2000) The stethoscope in the Emergency Department: a vector of infection? Epidemiol Infect 124:233237

45. Youngster I, Berkovitch M, Heyman E, Lazarovitch Z, Goldman M (2008) The stethoscope as a vector of infectious diseases in the paediatric division. Acta Paediatr 97:1253-1255

46. National Institute for Health and Care Excellence (NICE)(2012) Infection: Prevention and control of healthcareassociated infections in primary and community care. NICE clinical guideline 139

47. Burger A et al (2011) Bare below elbows: does this policy affect handwashing efficacy and reduce bacterial colonisation? Ann R Coll Surg Engl 93(1):13-16

48. Willis-Owen CA et al (2010) Effects of, bare below the elbows' policy on hand contamination of 92 hospital doctors in a district general hospital. J Hosp Infect 75(2):116-119

49. Hautemaniere A et al (2010) Factors determining poor practice in alcoholic gel hand rub technique in hospital workers. J Infect Public Health 3(1):25-34 
50. Kac G et al (2005) Microbiological evaluation of two hand hygiene procedures achieved by healthcare workers during routine patient care: a randomized study. J Hosp Infect 60(1):32-39

51. DIN EN 14126:2004-01 Schutzkleidung - Leistungsanforderungen und Prüfverfahren für Schutzkleidung gegen Infektionserreger. Beuth Verlag: Berlin

52. Sartori M, La Terra G, Aglietta M, Manzin A, Navino C, Verzetti G (1993) Transmission of hepatitis $C$ via blood splash into conjunctiva. Scand J Infect Dis 25(2):270-271

53. Hosoglu S, Celen MK, Akalin S, Geyik MF, Soyoral Y, Kara IH (2003) Transmission of hepatitis C by blood splash into conjunctiva in a nurse. Am J Infect Control 31(8):502-504

54. Centers for Disease Control and Prevention (CDC) (1987) Update: human immunodeficiency virus infections in health-care workers exposed to blood of infected patients. MMWR Morb Mortal Wkly Rep 36(19):285-289

55. Keijman J, Tjhie J, Olde Damink S, Alink M (2001) Unusual nosocomial transmission of Mycobacterium tuberculosis. Eur J Clin Microbiol Infect Dis 20(11):808-209

56. Goins W, Talbot HK, Talbot TR (2011) Health careacquired viral respiratory diseases. Infect Dis Clin North Am 25(1):227-244

57. Hall C (1981) Nosocomial viral respiratory infections: perennial weeds on pediatric wards. Am J Med 70:670-676

58. Bund/Länder-Arbeitsgemeinschaft Abfall (LAGA)(2015) Mitteilung der Bund/Länder-Arbeitsgemeinschaft Abfall (LAGA) 18. Vollzugshilfe zur Entsorgung von Abfällen aus Einrichtungen des Gesundheitsdienstes

59. Fijan S, Turk SS (2012) Hospital textiles, are they a possible vehicle for healthcare-associated infections? Int J Environ Res Public Health 9(9):3330 3343

60. Creamer $\mathrm{E}$, Humphreys $\mathrm{H}$ (2008) The contribution of beds to healthcare-associated infection: the importance of adequate decontamination. J Hosp Infect 69(1):8-23

61. Bloomfield SF, Exner M, Signorelli C, Scott EA (2013) Effectiveness of Laundering Processes Used in Domestic (home) Settings. International Scientific Forum on Home Hygiene (ISH), URL: http://www.ifhhomehygiene.org/review/effectiveness-laundering-processes-used-domestic-home-settings-2013

62. MEDLINE (Medical Literature Analysis and Retrieval System Online) Online-Zugriff bspw. über die Datenbank PubMed. http://www.ncbi.nlm.nih. gov/pubmed

63. Lebensmittelhygiene-Verordnung vom 8 . August 2007 (BGBI. I S. 1816, 1817), die zuletzt durch Artikel 1 der Verordnung vom 14. Juli 2010 (BGBI. I S. 929) geändert worden ist

64. Landers T, Abusalem S, Coty MB, Bingham J (2012) Patient-centered hand hygiene: the next step in infection prevention. Am J Infect Control 40(4 Suppl 1):11-17

65. Gudnadottir U, Fritz J, Zerbel S, Bernardo A, Sethi AK, Safdar N (2013) Reducing health care-associated infections: patients want to be engaged and learn about infection prevention. Am J Infect Control 41(11):955-958
66. Evans C, Hill JN, Guihan M, Chin A, Goldstein B, Richardson MS, Anderson V, Risa K, Kellie S, Cameron KA (2013) Implementing a patient education intervention about MRSA prevention and effect on knowledge and behavior in veterans with spinal cord injuries and disorders: a pilot randomized controlled trial. J Spinal Cord Med 37(2):152-161

67. Mielke M, Nassauer A, Robert Koch-Institut (Hrsg.) (2009) Herleitung von risikominimierenden, hier infektionspräventiven Maßnahmen in der Praxis. Bedeutung der Standardhygiene und ggf. ergänzender Maßnahmen zum Schutz von Patienten und Personal vor nosokomialen Infektionen. URL: https://www.rki.de/DE/Content/Infekt/Krankenhaushygiene/Erreger_ausgewaehlt/ Einleit_pdf

68. Kommission für Krankenhaushygiene und Infek tionsprävention (2009) Personelle und organisatorische Voraussetzungen zur Prävention nosokomialer Infektionen. Bundesgesundheitsbl 52:951-962

69. Nseir $S$ et al (2011) Risk of acquiring multidrugresistant Gram-negative bacilli from prior room occupants in the intensive care unit. Clin Microbiol Infect 17(8):1201-1208

70. Landelle C, Pagani L, Harbarth S (2013) Is patient isolation the single most important measure to prevent the spread of multidrug-resistant pathogens? Virulence 4(2):163-171

71. Centers for Disease Control and Prevention (CDC) (1997) Nosocomial hepatitis B virus infection associated with reusable fingerstick blood sampling devices - Ohio and New York City, 1996. MMWR Morb Mortal Wkly Rep 46(10):217-221

72. Wright S, Gerry JS, Busowski MT et al (2012) Gordonia bronchialis sternal wound infection in 3 patients following open heart surgery: intraoperative transmission from a healthcare worker. Infect Control Hosp Epidemiol 33(12):1238-1241

73. Feigin R, Baker CJ, Herwaldt LA, Lampe RM, Mason EO, Whitney SE (1982) Epidemic meningococcal disease in an elementary-school classroom. N Engl J Med 307(20):1255-1257

74. Dick $E$, Jennings LC, Mink KA, Wartgow CD, Inhorn SL (1987) Aerosol transmission of rhinovirus colds. J Infect Dis 156(3):442-448

75. Simonds A, Hanak A, Chatwin M, Morrell M, Hall A, Parker KH, Siggers JH, Dickinson RJ (2010) Evaluation of droplet dispersion during non-invasive ventilation, oxygen therapy, nebuliser treatment and chest physiotherapy in clinical practice: implications for management of pandemic influenza and other airborne infections. Health Technol Assess 14(46):131-172

76. Tang J, Li Y, Eames I, Chan PK, Ridgway GL (2006) Factors involved in the aerosol transmission of infection and control of ventilation in healthcare premises. J Hosp Infect 64:100-114

77. Macdonald TM, Langley JM, Mailman T, et al (2011) Serratia marcescens outbreak in a neonatal intensive care unit related to the exit port of an oscillator. Pediatr Crit Care Med 12(6):e:282286

78. Wong B, Lee N, Li Y, Chan PK, Qiu H, Luo Z, Lai RW, Ngai KL, Hui DS, Choi KW, Yu IT (2010) Possible role of aerosol transmission in a hospital outbreak of influenza. Clin Infect Dis 51(10):11761183

79. Tran K, Cimon K, Severn M, Pessoa-Silva CL, Conly $J$ (2012) Aerosol generating procedures and risk of transmission of acute respiratory infections to healthcare workers: a systematic review. PLoS One 7(4):e35797
80. Siegel J, Rhinehart E, Jackson M, Chiarello L, The Healthcare Infection Control Practices Advisory Committee (2007) 2007 Guideline for isolation precautions: preventing transmission of infectious agents in healthcare settings. Am J Infect Control 35(10 Suppl 2):S65-S164

81. Bloch A, Orenstein WA, Ewing WM et al (1985) Measles outbreak in a pediatric practice: airborne transmission in an office setting. Pediatrics 75(4):676-683

82. Coronado V, Beck-Sague CM, Hutton MD et al (1993) Transmission of multidrug-resistant Mycobacterium tuberculosis among persons with human immunodeficiency virus infection in an urban hospital: epidemiologic and restriction fragment length polymorphism analysis. J Infect Dis 168(4):1052-1055

83. Fernstrom A, Goldblatt M (2013) Aerobiology and its role in the transmission of infectious diseases. J Pathog 493960

84. Lindner J, Modrow S (2008) Human bocavirus - a novel parvovirus to infect humans. Intervirology 51(2):116-221

85. Boršo D, Löbermann M, Fritzsche $C$, Hemmer $C$, Führer A, Zettl U, Reisinger EC (2013) [Vaccinations in patients with immunodeficiency or immunosuppressive therapy]. Dtsch Med Wochenschr 138(4):145-150

86. Ständige Impfkommission (STIKO) (2014) Mitteilung der Ständigen Impfkommission am Robert Koch-Institut (RKI). Empfehlungen der Ständigen Impfkommission (STIKO) am Robert Koch-Institut/Stand: August 2014. Epidemiol Bulletin 34

87. Esolen L, Kilheeney KL (2013) A mandatory campaign to vaccinate health care workers against pertussis. Am J Infect Control 41(8):740-742

88. Maltezou H, Wicker S (2013) Measles in healthcare settings. Am J Infect Control 41(7):661-663

89. Banach D, Zhang C, Factor SH, Calfee DP (2013) Support for mandatory health care worker influenza vaccination among allied health professionals, technical staff, and medical students. Am J Infect Control 41(4):354-356

90. Adler A, Casper C, Boeckh M, Heath J, Zerr DM (2008) An outbreak of varicella with likely breakthrough disease in a population of pediatric cancer patients. Infect Control Hosp Epidemiol 29:866-870

91. Manley S, Mallinson H, Caswell M, Keenan R, Pizer B (2008) Chickenpox in varicella IgG positive patients: experience of a regional paediatric oncology centre. Pediatr Blood Cancer 51:540-542

92. Matsuzaki A, Suminoe A, Koga Y et al (2008) Fatal visceral varicella-zoster virus infection without skin involvement in a child with acute lymphoblastic leukemia. Pediatr Hematol Oncol 25:237242

93. Rau R, Fitzhugh CD, Baird K et al (2008) Triad of severe abdominal pain, inappropriate antidiuretic hormone secretion, and disseminated varicella-zoster virus infection preceding cutaneous manifestations after hematopoietic stem cell transplantation: utility of PCR for early recognition and therapy. Pediatr Infect Dis J 27:265-268

94. Mutterschutzgesetz in der Fassung der Bekanntmachung vom 20. Juni 2002 (BGBI. I S. 2318), das zuletzt durch Artikel 6 des Gesetzes vom 23. Oktober 2012 (BGBI. I S. 2246) geändert worden ist

95. Tai YJ et al (2013) Nasal carriage of Staphylococcus aureus in patients undergoing Mohs micrographic surgery is an important risk factor for postoperative surgical site infection: a prospective randomised study. Australas J Dermatol 54(2):109-114 
96. van de Glind I, de Roode S, Goossensen A (2007) Do patients in hospitals benefit from single rooms? A literature review. Health Policy 84(23):153-161

97. Dettenkofer M, Seegers S, Antes G, Motschall E, Schumacher M, Daschner FD (2004) Does the architecture of hospital facilities influence nosocomial infection rates? A systematic review. Infect Control Hosp Epidemiol 25(1):21-25

98. Teltsch D, Hanley J, Loo V, Goldberg P, Gursahaney A, Buckeridge DL (2011) Infection acquisition following intensive care unit room privatization. Arch Intern Med 171(1):32-38

99. Haertel C, Simon A, Geffers C et al (2013) Nosokomiale Infektionen bei Frühgeborenen - Umsetzung der KRINKO-Empfehlungen im Deutschen Frühgeborenennetzwerk. Monatsschr Kinderheilkd 161:27-33

100. Department of Health (UK) (2013) In-patient care. Health Building Note 04-01: adult in-patient facilities

101. Dreller $S$, Jatzwauk L, Nassauer A, Paskiewicz $P$, Tobys HU, Rüden H (2006) Zur Frage des geeigneten Atemschutzes vor luftübertragenen Infektionserregern. Gefahrstoffe Reinhalt Luft 66(12):14-24

102. Ziegler R, Just HM, Castell $S$, Diel R, Gastmeier P, Haas W, Hauer B, Loytved G, Mielke M, Moser I, Nienhaus A, Richter E, Rüden H, Rüsch-Gerdes S, Schaberg T, Wischnewski N, Loddenkemper R (2012) Infektionsprävention bei Tuberkulose. Empfehlungen des DZK. Gesundheitswesen 74(6):337-350

103. Arbeitsmedizinische Regeln (AMR) Nr. 14.2 Einteilung von Atemschutzgeräten in Gruppen, in Bek. D. BMAS v. 26.03.2014

104. Deutsche Gesetzliche Unfallversicherung(DGVU) (2010) Handlungsanleitung für die arbeitsmedizinische Vorsorge nach dem DGUV Grundsatz G26 „Atemschutzgeräte“. BGI/GUV-I 504-526

105. Verordnung über Sicherheit und Gesundheitsschutz bei Tätigkeiten mit Biologischen Arbeitsstoffen, Biostoffverordnung vom 15. Juli 2013 (BGBI. IS. 2514)

106. Jefferson T, Foxlee R, Del Mar C, Dooley L, Ferroni E, Hewak B, Prabhala A, Nair S, Rivetti A (2008) Physical interventions to interrupt or reduce the spread of respiratory viruses: systematic review. BMJ 336(7635):77-80

107. Bin-Reza F, Lopez Chavarrias V, Nicoll A, Chamberland ME (2012) The use of masks and respirators to prevent transmission of influenza: a systematic review of the scientific evidence. Influenza Other Respir Viruses 6(4):257-267

108. BischoffW, Reid T, Russell GB, Peters TR (2011) Transocular entry of seasonal influenza-attenuated virus aerosols and the efficacy of $n 95$ respirators, surgical masks, and eye protection in humans. J Infect Dis 204:193-199

109. Lindsley W, Blachere FM, Davis KA, Pearce TA, Fisher MA, Khakoo R, Davis SM, Rogers ME, Thewlis RE, Posada JA, Redrow JB, Celik IB, Chen BT, Beezhold DH (2010) Distribution of airborne influenza virus and respiratory syncytial virus in an urgent care medical clinic. Clin Infect Dis 50:693-698

110. Noti J, Lindsley WG, Blachere FM, Cao G, Kashon ML, Thewlis RE, McMillen CM, King WP, Szalajda JV, Beezhold DH (2012) Detection of infectious influenza virus in cough aerosols generated in a simulated patient examination room. Clin Infect Dis 54:1569-1577
111. Larson $E$, Liverman CT, Institute of Medicine of the National Academies (2010) Preventing transmission of pandemic influenza and other respiratory viral infectious diseases update 2010 The National Academies Press, Washington D.C. (http://www.nap.edu)

112. Resch B, Manzoni P, Lanari M (2009) Severe respiratory syncytial virus (RSV) infection in infants with neuromuscular diseases and immune deficiency syndromes. Paediatr Respir Rev 10(3):148-153

113. Lai $C$, Wang $Y H$, Wu $C Y$, Hung $C H$, Jiang DD, Wu FT (2013) A norovirus outbreak in a nursing home: norovirus shedding time associated with age. J Clin Virol 56(2):96-101

114. Doshi M, Woodwell S, Kelleher K, Mangan K, Axelrod P (2013) An outbreak of norovirus infection in a bone marrow transplant unit. Am J Infect Control 41(9):820-823

115. Geis S, Prifert C, Weissbrich B, Lehners N, Egerer G, Eisenbach C, Buchholz U, Aichinger E, Dreger P, Neben K, Burkhardt U, Ho AD, Krausslich HG, Heeg K, Schnitzler P (2013) Molecular characterization of a respiratory syncytial virus outbreak in a hematology unit in Heidelberg, Germany. J Clin Microbiol 51:155-162

116. van den Broek P, Colville A, Coignard B, Daha $\mathrm{T}$, Debast S, Duerden $\mathrm{Bl}$, van den Hof S, van der Kooi T, Maarleveld HJ, Nagy E, Notermans DW, O'Driscoll J, Patel B, Stone S, Wiuff C (2008) Infection control measures to limit the spread of Clostridium difficile. Clin Microbiol Infect 14(5):2-20

117. Vonberg R, Kuijper EJ, Wilcox MH, Barbut F, Tüll $P$, Gastmeier $P$ (2008) Infection control measures to limit the spread of Clostridium difficile. Clin Microbiol Infect 14:2-20

118. Ausschuss für Biologische Arbeitsstoffe (2012) Beschluss 609 - Arbeitsschutz beim Auftreten einer nicht ausreichend impfpräventablen humanen Influenza. 2012: GMBI. Nr. 26 vom 18. Juni 2012, S 470-479

119. Robert Koch-Institut (2004) Prüfung und Deklaration der Wirksamkeit von Desinfektionsmitteln gegen Viren. Bundesgesundheitsblatt Gesundheitsforschung Gesundheitsschutz 47(1):62-66 